\title{
Glutaredoxin1 Diminishes Amyloid Beta-Mediated Oxidation of F-Actin and Reverses Cognitive Deficits in an Alzheimer's Disease Mouse Model
}

\author{
Reddy Peera Kommaddi, ${ }^{1}$ Deepika Singh Tomar, ${ }^{1}$ Smitha Karunakaran, ${ }^{1}$ Deepti Bapat, ${ }^{1}$ Siddharth Nanguneri, ${ }^{1}$ \\ Ajit Ray, Bernard L. Schneider, ${ }^{2}$ Deepak Nair, and Vijayalakshmi Ravindranath ${ }^{1,3}$
}

\begin{abstract}
Aims: Reactive oxygen species (ROS) generated during Alzheimer's disease (AD) pathogenesis through multiple sources are implicated in synaptic pathology observed in the disease. We have previously shown Factin disassembly in dendritic spines in early AD (34). The actin cytoskeleton can be oxidatively modified resulting in altered F-actin dynamics. Therefore, we investigated whether disruption of redox signaling could contribute to actin network disassembly and downstream effects in the amyloid precursor protein/presenilin-1 double transgenic (APP/PS1) mouse model of AD.

Results: Synaptosomal preparations from 1-month-old APP/PS1 mice showed an increase in ROS levels, coupled with a decrease in the reduced form of F-actin and increase in glutathionylated synaptosomal actin. Furthermore, synaptic glutaredoxin 1 (Grx1) and thioredoxin levels were found to be lowered. Overexpressing Grx1 in the brains of these mice not only reversed F-actin loss seen in APP/PS1 mice but also restored memory recall after contextual fear conditioning. F-actin levels and F-actin nanoarchitecture in spines were also stabilized by Grx1 overexpression in APP/PS1 primary cortical neurons, indicating that glutathionylation of Factin is a critical event in early pathogenesis of $\mathrm{AD}$, which leads to spine loss.

Innovation: Loss of thiol/disulfide oxidoreductases in the synapse along with increase in ROS can render Factin nanoarchitecture susceptible to oxidative modifications in AD.

Conclusions: Our findings provide novel evidence that altered redox signaling in the form of S-glutathionylation and reduced Grx1 levels can lead to synaptic dysfunction during AD pathogenesis by directly disrupting the Factin nanoarchitecture in spines. Increasing Grx1 levels is a potential target for novel disease-modifying therapies for AD. Antioxid. Redox Signal. 31, 1321-1338.
\end{abstract}

Keywords: Grx1, oxidation, reactive oxygen species, neurodegenerative disease, cytoskeleton, cognition

\section{Introduction}

A LZHEIMER'S DISEASE (AD) is one of the most common types of dementia in aging individuals that impair memory and higher cognitive functions. Pathological hallmarks of $\mathrm{AD}$ include aggregation of $\beta$-amyloid seen as extracellular plaques and neurofibrillary tangles due to hyperphosphorylated Tau $(24,34,54,65)$. Soluble amyloid beta $(\mathrm{A} \beta)$ oligomers have been shown to be the most potent contributors to AD pathogenesis $(36,67)$, and synaptic dysfunction accompanied by loss of dendritic spines occurs in the early stages of disease $(27,55)$.

Dendritic spines are small membranous protrusions located along the neuritic shaft serving as specialized zones for excitatory postsynaptic machinery. The shape of these spines is determined by the F-actin cytoarchitecture, which undergoes treadmilling and restructuring particularly during synaptic plasticity $(13,18,29)$. Actin filaments (F-actin) are formed by

\footnotetext{
${ }^{1}$ Centre for Neuroscience, Indian Institute of Science, Bangalore, India.

${ }^{2}$ Brain Mind Institute, Ecole Polytechnique Fédérale de Lausanne, Lausanne, Switzerland.

${ }^{3}$ Centre for Brain Research, Indian Institute of Science, Bangalore, India.
} 


\section{Innovation}

Maintenance of F-actin and G-actin homeostasis in dendritic spines is critical for synaptic function and extremely important for synaptic plasticity. In this study, we show that oxidative modification of F-actin leads to recall deficits after contextual fear conditioning and overexpression of glutaredoxin 1 (Grx1) can rescue these deficits by restoring F-actin dynamics in dendritic spines. The present study demonstrates for the first time the role of Grx 1 in maintaining F-actin dynamics in dendritic spines and that its disruption occurs early in Alzheimer's disease (AD) pathogenesis. Thus, therapeutic strategies targeted to augmenting Grx1 may help to reverse the pathological cascade in early AD.

polymerization of globular actin monomer (G-actin), and the degree of actin polymerization ( $\mathrm{G}$-actin/F-actin ratio) is critical to this process. Dysregulation of actin polymerization affects both structure and function of dendritic spines in $\mathrm{AD}$ (34). Actin modulators, such as Cdc42 (39), mTORC2 (30), LIMK1 (26), actin-depolymerizing factor/cofilin (7), and drebrin (34), are involved in F-actin assembly/disassembly in AD. Emerging evidence indicates posttranslational modifications of actin, such as acetylation (48), methylation (61), nitrosylation (22), phosphorylation (8), and redox-mediated thiol modifications [reviewed in ref. (61)], influence actin polymerization. Oxidative modification of actin can potentially change actin assembly, thereby altering the G-actin/F-actin ratio $(15,31,32)$.

Perturbations in protein thiol (PrSH) status, oxidative stress, and synaptic dysfunction are major contributors in aging-related pathology, and during AD pathogenesis (62). Increased production of reactive oxygen species (ROS) and reactive nitrogen species, and dysfunction of antioxidant enzymes have been shown in several studies to contribute significantly to AD pathogenesis $(9,10,14,37)$. Proteins modified by oxidation and nitrosylation have been identified by redox proteomics in amnestic mild cognitive impairment (aMCI), preclinical AD, and late-stage AD brains $(16,59)$. Oxidative modification of several proteins, such as aldolase, glyceraldehyde 3-phosphate dehydrogenase, $\alpha$-enolase, phosphoglycerate mutase 1 (9), aconitase, creatine kinase, and ATP-synthase, has been demonstrated in aMCI and AD brains $(16,60)$. As seen in human tissue, $\mathrm{A} \beta$-induced oxidative stress, ROS production, and lipid peroxidation have been shown in AD mouse models $(1,45)$.

The oxidation status of thiols is reversibly regulated by a group of enzymes called PrSH/disulfide oxidoreductases. These proteins catalyze different disulfide interchange reactions, including conversion of glutathionylated proteins (PrSSG) to PrSHs (50). One such enzyme, glutaredoxin 1 (also known as thioltransferase; Grx1), acts as a glutathione (GSH)-disulfide transhydrogenase and specifically and efficiently reduces glutathionylated proteins (PrSSG) to $\mathrm{PrSH}$ (50), but may reverse the direction based on cellular redox potential (52). Reversible modifications, such as glutathionylation, can prevent $\mathrm{PrSH}$ from undergoing irreversible oxidation, and likely inactivation, during oxidative stress. Indeed, glutathionylation of p53, a key stress/death signaling protein, is increased in AD samples compared with controls
(17). Even the levels of thiol/disulfide oxidoreductases, such as thioredoxin (Trx) and glutaredoxin, are shown to be altered in $\mathrm{AD}$ patients compared with controls $(4,6)$. Interestingly, actin polymerization can be altered by glutathionylation at its Cys-374 residue (15). Therefore, we wanted to examine whether $\mathrm{A} \beta$-induced oxidative stress results in oxidation of actin, specifically glutathionylation leading to F-actin loss in an AD mouse model. Finally, we tested if we can prevent this loss through overexpression of Grx1 in the mouse model of AD.

\section{Results}

\section{ROS levels are increased in amyloid precursor protein/ presenilin-1 double transgenic mice}

$\mathrm{A} \beta$ induces a large amount of ROS in human neuroblastoma cells, in vitro (66), AD human brains (12), and in AD mouse models $(1,25)$. To examine the role of ROS-induced redox signaling in synaptosomes, we first determined the levels of ROS in synaptosomes and postnuclear supernatant (PNS) isolated from 1 (adolescent [ADL])- and 9 (middleaged [MA])-month-old wild-type (WT) and amyloid precursor protein/presenilin-1 double transgenic (APP/PS1) mice using a cell permeant indicator for ROS, 2', 7'dichlorodihydrofluorescein diacetate $\left(\mathrm{H}_{2} \mathrm{DCFDA}\right)$. Rate of increase in $2^{\prime}, 7^{\prime}$-dichlorofluorescein (DCF) fluorescence with time is seen in synaptosomes from both ADL and MA WT, and APP/PS1 mice (Fig. 1A, D). ROS levels are significantly increased in both synaptosomes and PNS from 1- and 9month-old APP/PS1 mice when compared with WT mice (Fig. 1B, C, E, and F). Measurement of ROS by the $\mathrm{H}_{2} \mathrm{DCF}-$ $\mathrm{DA}$ method is indirect as it will recognize a broad range of oxygen free radicals and other oxidants. Thus, ROS data provided herein are only indicative of increased oxidative stress in APP/PS1 samples relative to WT.

\section{Reduced form of F-actin is diminished in synaptosomes of APP/PS1 mice}

Actin is highly susceptible to oxidative posttranslational modifications of its amino acid residues and several lines of evidence indicate that oxidation leads to decreased polymerization rate of $\mathrm{G}$-actin to F-actin $(15,19)$. However, the physiological importance of actin oxidation at the synapse is still poorly understood. Therefore, we examined if synaptosomal F-actin was oxidatively modified, which could potentially impact $\mathrm{F}$-actin dynamics and alter synaptic function. G-actin/F-actin fractions were isolated from cortical synaptosomes from WT and APP/PS1 male mice (ADL and MA). We then isolated G-actin and F-actin fractions from PNS, and all fractions were subjected to derivatization with the thiolreactive reagent 4 -acetamido- $4^{\prime}$-maleimidylstilbene-2, $2^{\prime}$ disulfonic acid, disodium salt (AMS). Intriguingly, while synaptosomal fractions showed significant loss in reduced forms of F-actin and total actin in APP/PS1 mice of all ages examined (Fig. 2A, B, D, and E; respectively, and Supplementary Figs. S5, S6, S8, and S9), no differences were found in the relative levels of synaptosomal-reduced form of G-actin between WT and APP/PS1 mice at either ages (Fig. 2C, F, respectively, and Supplementary Figs. S7 and S10). Furthermore, reduced forms of F-actin, G-actin, or total actin levels were not altered in PNS (Fig. 3A-F, respectively, 
FIG. 1. ROS levels are increased in cortical synaptosomes and PNS of APP/PS1 mice. (A, D) Rate of DCF fluorescence was measured from synaptosomes of 1 (ADL)- and 9-month (MA)-old WT and APP/PS1 mice. (B, E) Synaptosomes and (C, F) postnuclear supernatants were incubated with $10 \mu M \mathrm{H}_{2}$ DCFDA in 0.1 $M$ phosphate buffer ( $\mathrm{pH}$ 7.4) for $5 \mathrm{~min}$ at room temperature. Samples were then analyzed on a fluorescence plate reader using excitation at $488 \mathrm{~nm}$ and emission at $525 \mathrm{~nm}$ every $30 \mathrm{~s}$ for $90 \mathrm{~min}$. Statistical analysis: unpaired, twotailed Mann-Whitney U test; WT versus APP/PS1; " $p$ " values are indicated within the histograms. Results are represented as mean \pm SEM ( $n=4-8$ per group). (A, D) For some points, the error bars are shorter than the height of the symbol. In these cases, GraphPad Prism does not display the error bars. ADL, adolescent; APP/PS1, amyloid precursor protein/presenilin-1 double transgenic; DCF 2',7'dichlorofluorescein; $\mathrm{H}_{2} \mathrm{DCFDA}$, $2^{\prime}, 7^{\prime}$-dichlorodihydrofluorescein diacetate; MA, middle aged; PNS, postnuclear supernatant; ROS, reactive oxygen species; SEM, standard error of mean; WT, wild type.
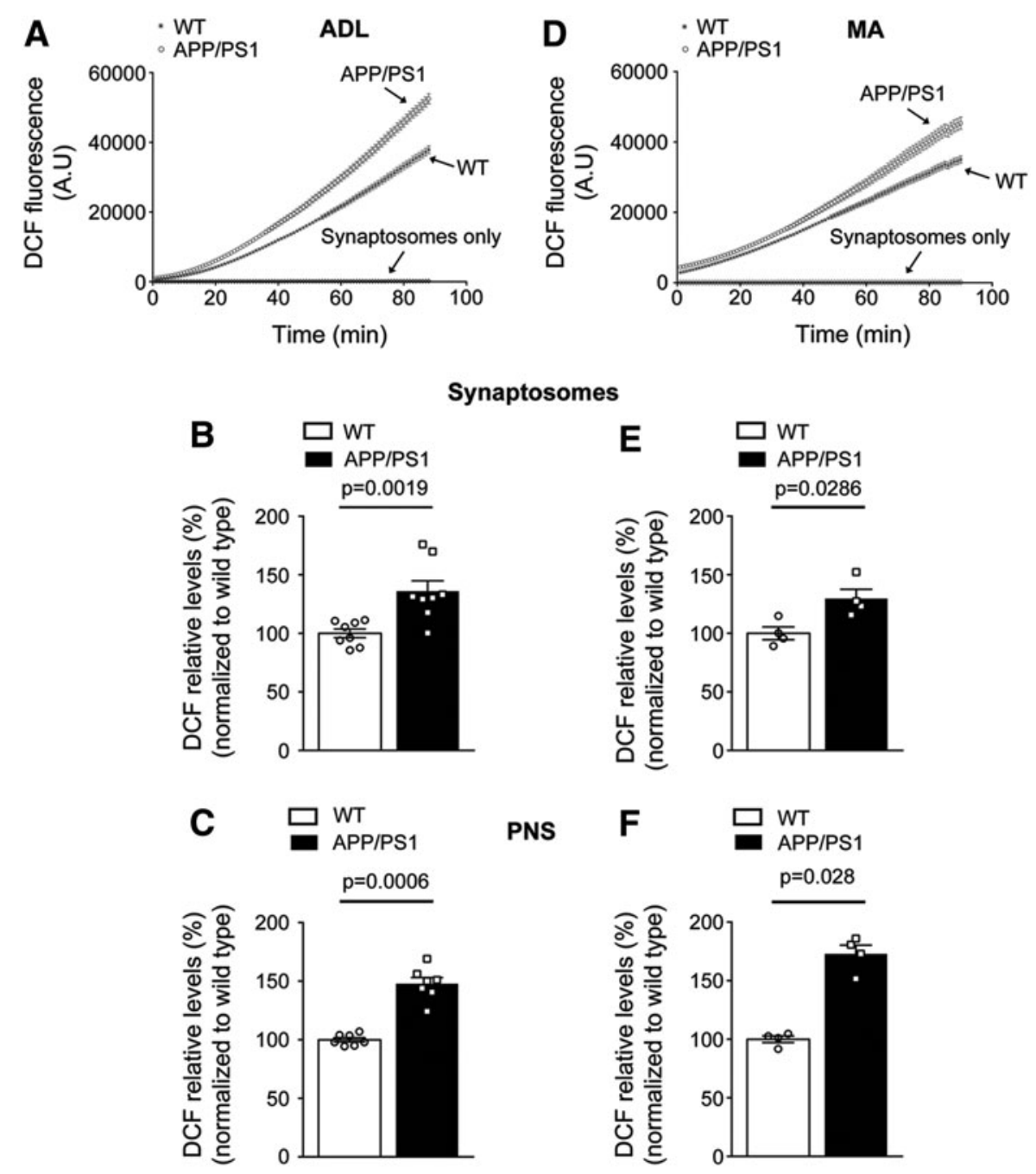

and Supplementary Figs. S15-S20). The synaptic proteins synaptosomal-associated protein $29 \mathrm{kDa}$ (SNAP29) and $\alpha$-synuclein were used as loading controls for F-actin fractions, synaptosomes, and G-actin fractions in redox blots since SNAP2 2 and $\alpha$-synuclein have no cysteine residues and hence cannot be derivatized by AMS. Thus, the amounts of SNAP29 or $\alpha$-synuclein measured in redox blots are unaffected by cysteine oxidation. We also separately assessed the total levels of these proteins in synaptosomes from WT and APP/PS1 mice (ADL and MA), and observed no change in the total SNAP29 (Supplementary Figs. S1A, B, S13, and S14) or $\alpha$-synuclein levels (data not shown). The immunoblots were also validated using polyclonal actin antibody (Supplementary Fig. S37). These data demonstrate that synaptosomal F-actin undergoes oxidative modification of cysteine residue(s).

We recently showed that synaptosomal F-actin levels were decreased in the cerebral cortex of APP/PS1 mice as early as 1 month of age (34). In this report, we also reproduce our previous results demonstrating that ADL APP/PS1 mice show significant decrease in synaptosomal F-actin levels in comparison with WT (Fig. 2G and Supplementary Fig. S11). In contrast, G-actin levels were significantly increased in synaptosomes from APP/PS1 mice during adolescence (Fig. 2H and Supplementary Fig. S12). Our previous and current results indicate that perturbation of synaptosomal G-actin or F-actin levels (G/F equilibrium) could have profound effects on the cytoskeletal architecture at the postsynaptic density and dendritic spine morphology, and thus impact synapse function. Therefore, selective loss of F-actin, as observed in ADL AD transgenic mice, before the appearance of overt pathological features could be a major contributing factor to synaptic deficits manifesting in these mice at later stages.

\section{S-glutathionylation of F-actin, G-actin, and total actin is increased in synaptosomes of APP/PS1 mice}

Protein S-glutathionylation is a dynamic reversible process (15) and is a regulatory switch affecting the function of numerous proteins, including actin, Akt1, protein tyrosine kinases, and phosphatases. Actin glutathionylation can occur on a critical cysteine, Cys374, inhibiting the rate of polymerization from G-actin to F-actin $(15,35,53)$. Nevertheless, whether actin glutathionylation is critical for actin-mediated synaptic functions under physiological condition remains unknown. To test this, lysates from cortical synaptosomes, along with synaptosomal F-actin fractions and G-actin fractions prepared from ADL mice (WT and APP/PS1), were immunoprecipitated using an anti-GSH antibody, followed by detection of actin by immunoblotting. Our results show that S-glutathionylation increased not only in synaptosomal total actin but also in F-actin 

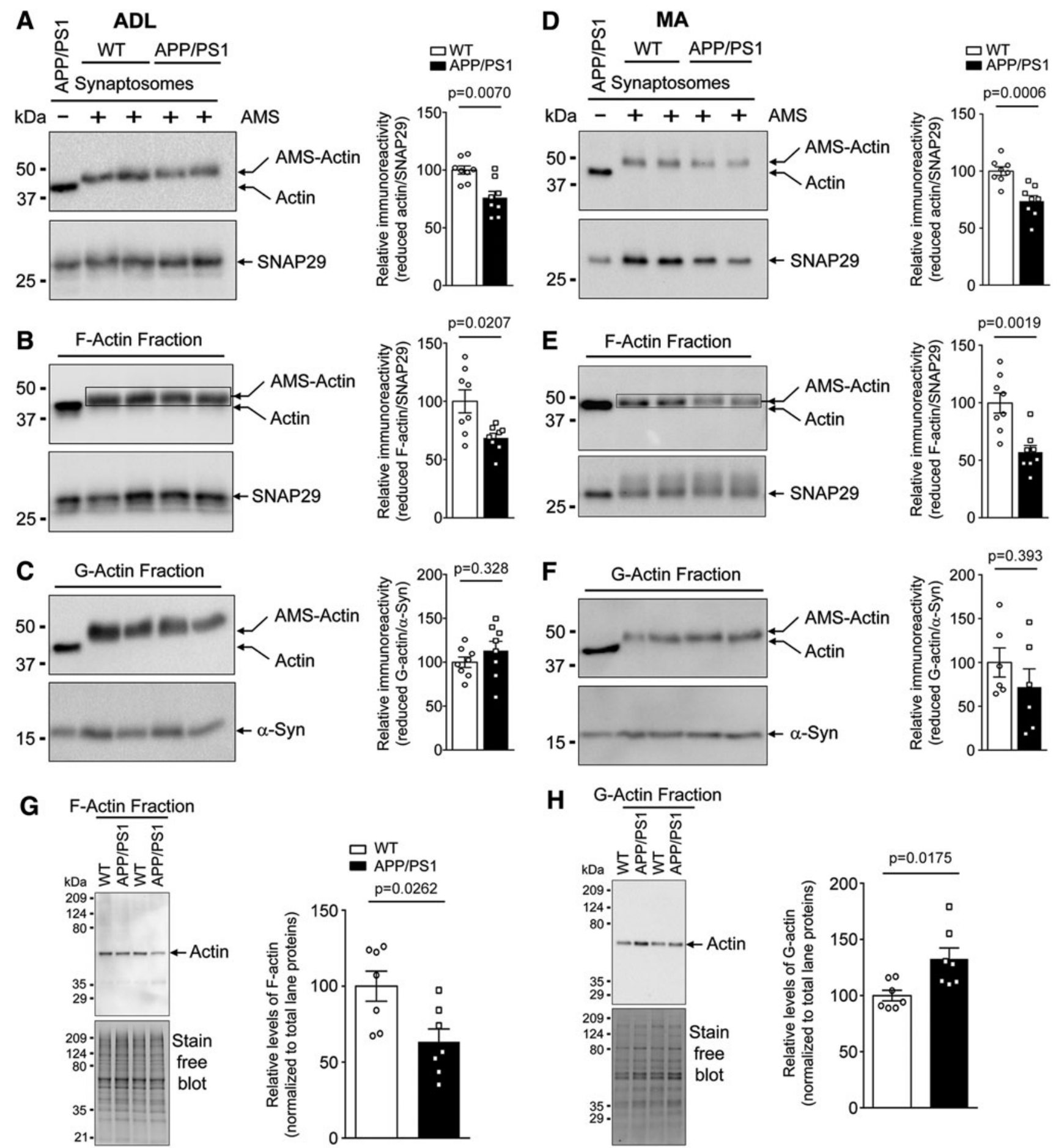

FIG. 2. Reduced total actin and F-actin levels are decreased in synaptosomes from the cerebral cortex of APP/PS1 mice. Synaptosomes and F-actin and G-actin fractions (isolated from synaptosomes) from ADL [(A-C); respectively] and MA [(D-F) respectively] WT and APP/PS1 mice brain cortex samples were subjected to SDS-PAGE and immunoblotting without or after treatment with the thiol alkylating agent, AMS. Densitometric analysis for AMS G-actin and AMS F-actin levels was normalized to $\alpha$-synuclein and SNAP29. Statistical analysis: unpaired, two-tailed Mann-Whitney U test. WT versus APP/PS1; " $p$ " values are indicated above the histograms. $p>0.05$ is considered no significance. Results are expressed as mean \pm SEM of 6-8 animals per group. All full-length unedited immunoblots for (A-F) are represented in Supplementary Figures S5-S10. Synaptosomal F-actin levels are decreased in the cerebral cortex of 1-month-old APP/PS1 mice. G-actin and F-actin fractions were isolated from synaptosomes of ADL (1-month) WT and APP/PS1 mouse brain cortex samples. $(\mathbf{G}, \mathbf{H})$ Levels of F-actin and G-actin were analyzed by immunoblotting. Densitometric analyses for actin levels were normalized to total lane proteins quantified by fluorescence of TGX stain-free blots. Results are represented as mean \pm SEM of seven animals per group. Statistical analysis: " $p$ " values for WT versus APP/PS1 are indicated above the histograms. Full-length unedited stainfree blots and immunoblots for $(\mathbf{G}, \mathbf{H})$ are represented in Supplementary Figures S11-S12. AMS, 4-acetamido-4'maleimidylstilbene-2,2'-disulfonic acid, disodium salt; SDS-PAGE, sodium dodecyl sulfate/polyacrylamide gel electrophoresis; SNAP29, synaptosomal-associated protein $29 \mathrm{kDa}$; TGX, tris-glycine-extended. 


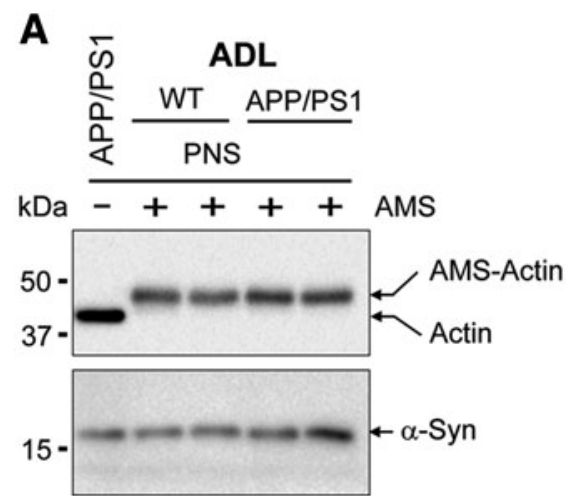

B

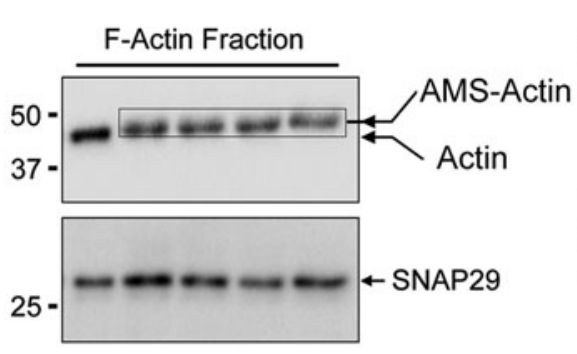

C

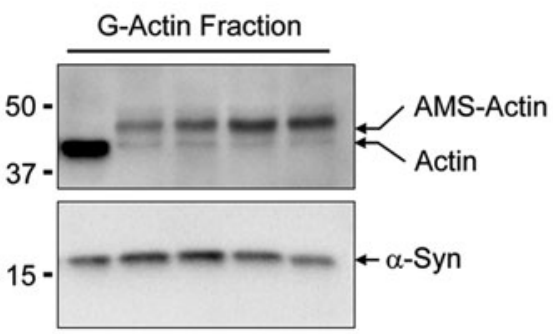

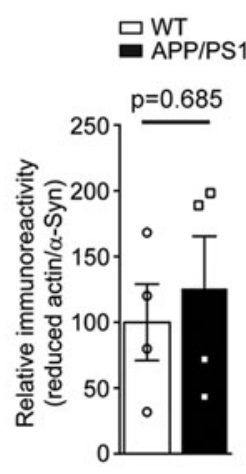
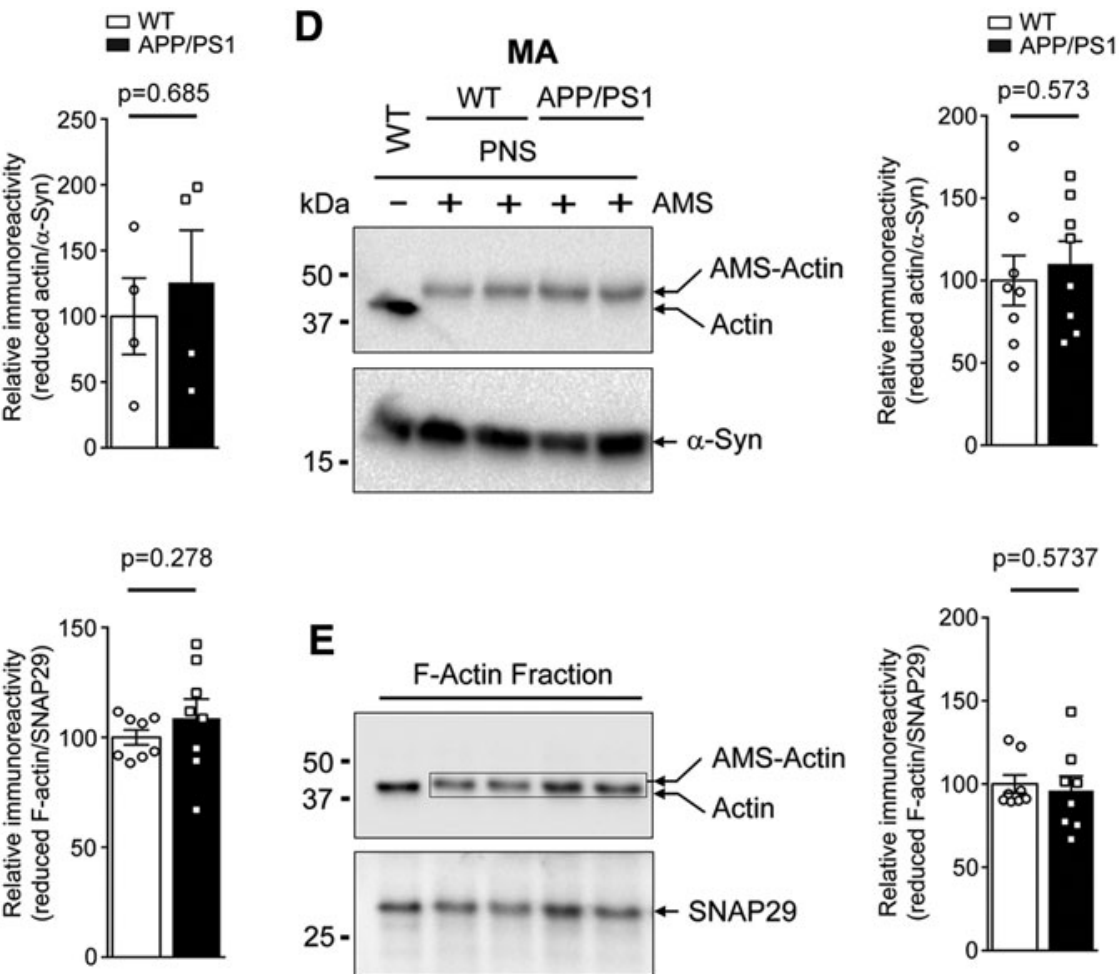

E
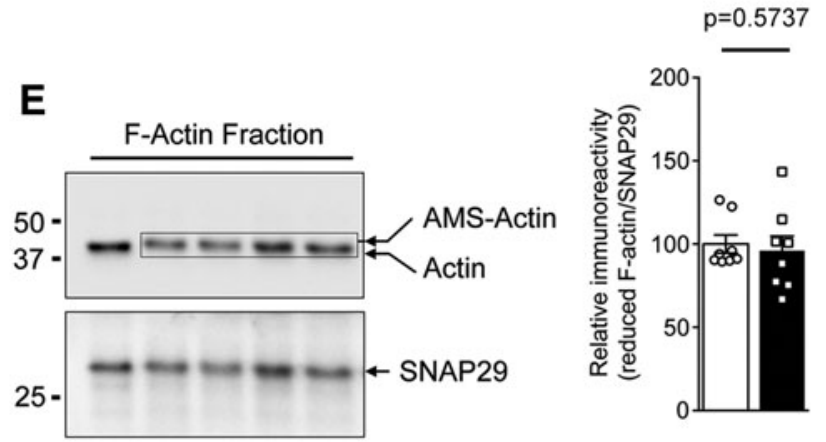

$\mathrm{p}=0.393$

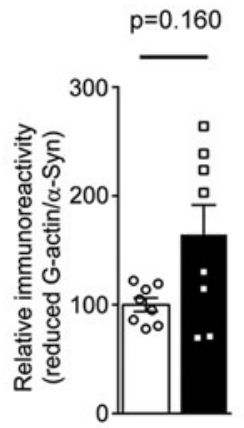

$\mathbf{F}$

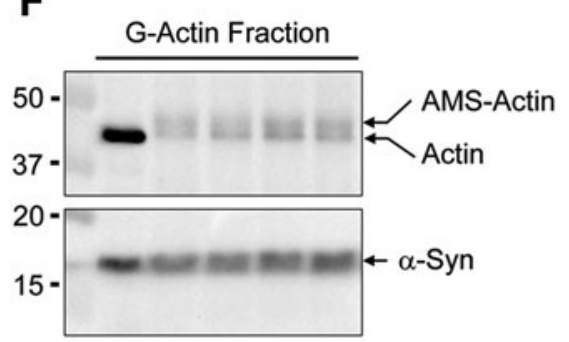

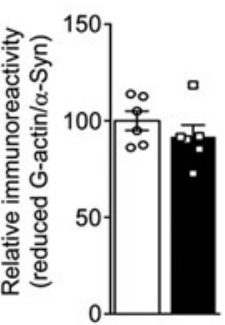

FIG. 3. Levels of reduced forms of total actin and F-actin in PNS from the cerebral cortex of APP/PS1 mice were unaltered compared with WT. PNS and F-actin and G-actin fractions (isolated from PNS) from ADL [(A, B, and C); respectively] and MA [(D, E, and F) respectively] WT and APP/PS1 mice brain cortex samples were subjected to SDSPAGE and immunoblotting without or after treatment with the thiol alkylating agent, AMS. Densitometric analysis for AMS G-actin and AMS F-actin levels is normalized to $\alpha$-synuclein and SNAP29. Results are expressed as mean \pm SEM of 4-8 animals per group. Unpaired, two-tailed Mann-Whitney U test. WT versus APP/PS1; " $p$ " values are mentioned above the histograms, respectively. $p>0.05$ is considered no significance. All full-length unedited immunoblots for (A-F) are represented in Supplementary Figures S15-S20.

and G-actin fractions isolated from synaptosomes (Fig. 4A-C and Supplementary Figs. S21-S26). Thus, we can conclude that synaptic F-actin and G-actin can be glutathionylated during oxidative stress generated during early pathogenesis of AD.

\section{Synaptosomal Grx1 and Trx levels are decreased in cerebral cortex of APP/PS1 mice}

Thiol/disulfide oxidoreductases, such as Grx1 and Trx, efficiently reduce S-PrSSG to PrSHs. Grx1 has been implicated in sulfhydryl homeostasis, redox regulation, iron metabolism, posttranslational modifications, and defense against oxidative stress (43). Thus, we tried to determine whether increased actin glutathionylation in synaptosomes could be due to loss of Grx1 and/or Trx in APP/PS1 mice. To examine this, we measured the levels of Grx1 and Trx in synaptosomes isolated from ADL and MA WT and APP/PS1 mice. Remarkably, ADL APP/PS1 mice showed a significant decrease in synaptosomal Grx1 and Trx levels, and this continued as the mice aged (Fig. 5A-D and Supplementary Figs. S27-S30). Therefore, we conclude that reduction of Grx1 and Trx levels in synaptic compartments could lead to increased glutathionylation of actin, which may then affect actin polymerization in APP/PS1 mice.

\section{Biochemical characterization of adeno-associated virus 6-Grx1 virus}

We optimized the adeno-associated virus (AAV) 6-Grx1 virus titer by transducing into primary cortical neurons and assessed the mRNA levels of Grx1 by quantitative real-time 

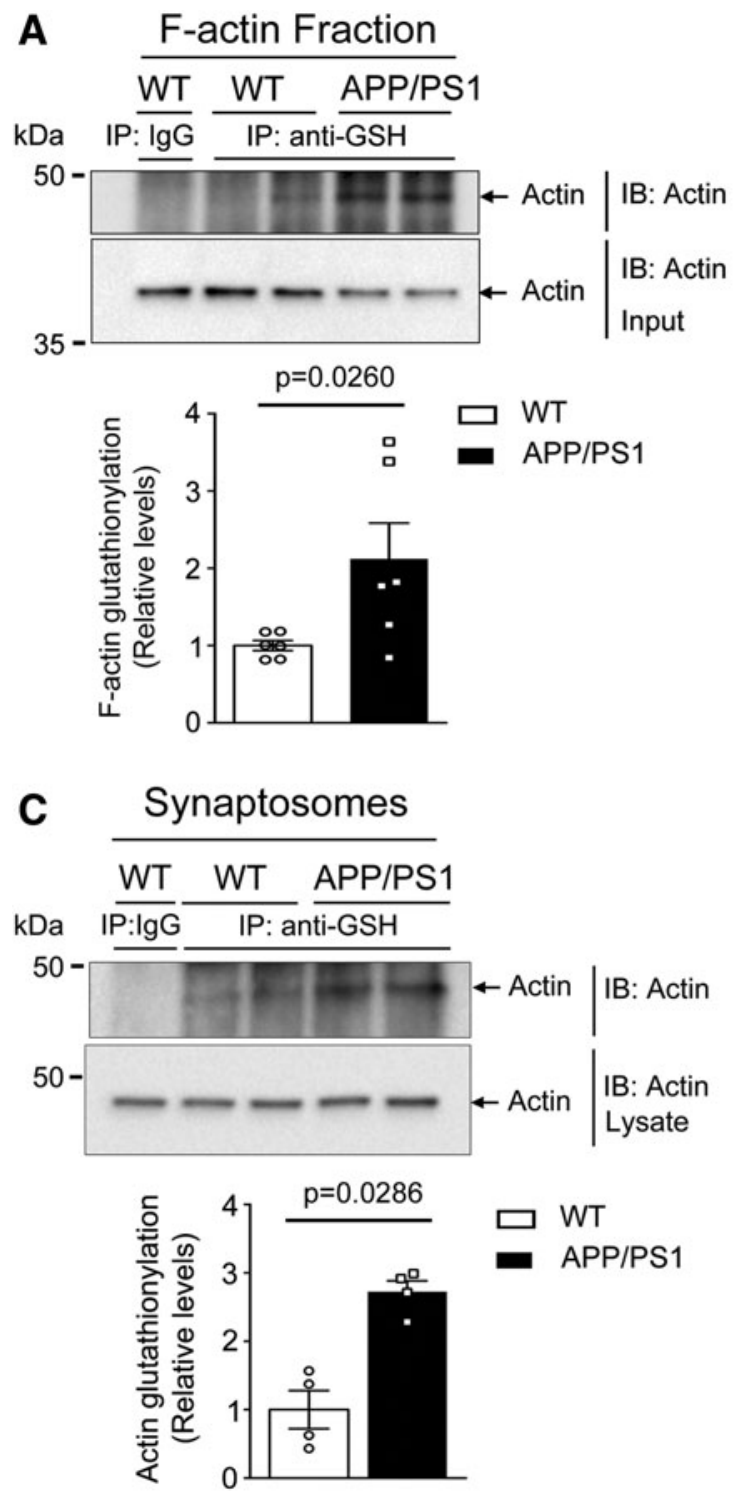

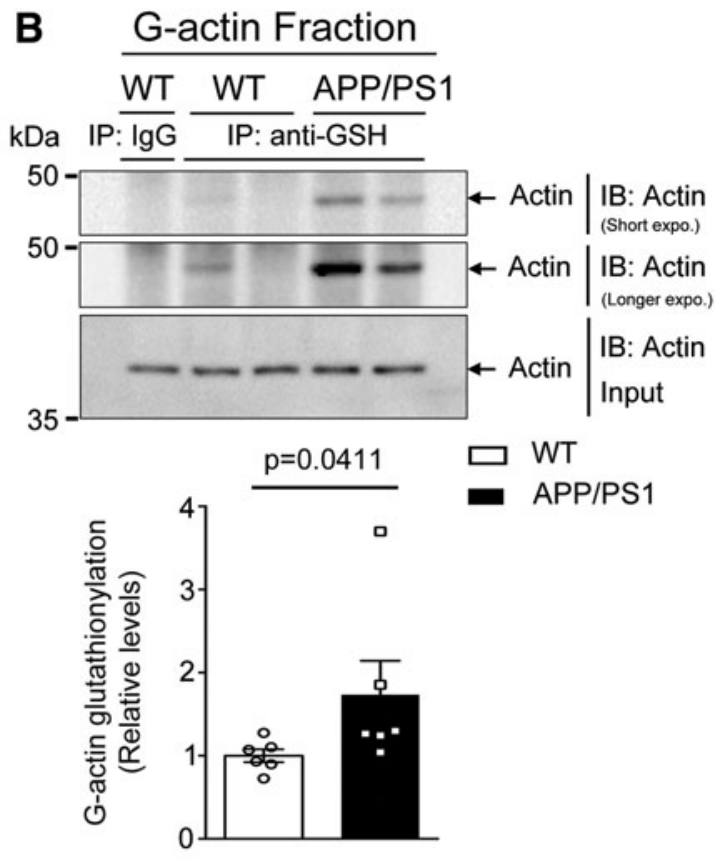

D

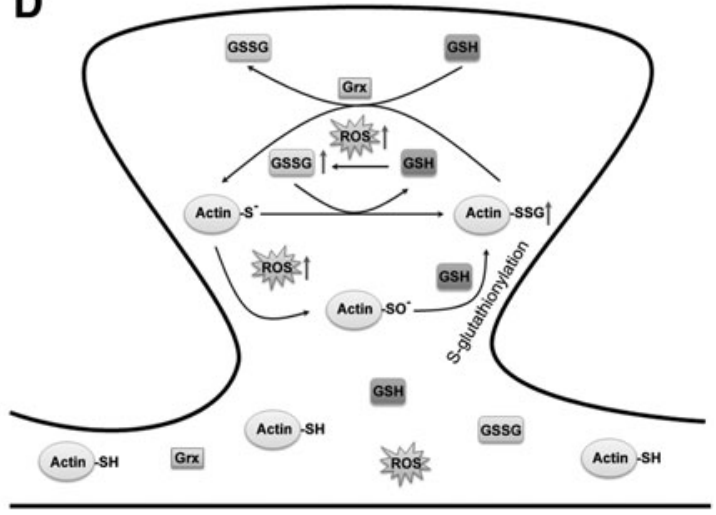

FIG. 4. F-actin, G-actin, and total actin S-glutathionylation is increased in synaptosomes from the cerebral cortex of APP/PS1 mice. S-PrSSG from F-actin fractions (A), G-actin fractions (B), and lysed synaptosomes (C) from ADL mice were immunoprecipitated with the anti-GSH monoclonal antibody. The immunoprecipitates (IP) were separated by $10 \%$ SDS-PAGE under reducing (+DTT) conditions and IB with antibody specific to actin. Full-length unedited immunoblots for (A-C) are represented in Supplementary Figures S21-S26. Densitometric analysis for F-actin glutathionylation [(A); histogram], G-actin glutathionylation [(B); histogram], and actin glutathionylation [(C); histogram] levels is normalized to WT controls. Results are represented as mean \pm SEM of 4-6 animals per group. Statistical analysis: WT versus APP/PS1 (unpaired, two-tailed Mann-Whitney U test). (D) Scheme of S-glutathionylation/deglutathionylation in the dendritic spine. DTT, dithiothreitol; GSH, glutathione; IB, immunoblot; IP, immunoprecipitation; PrSSG, glutathionylated proteins.

polymerase chain reaction (Fig. 6A) and immunoblotting (Fig. 6B, C, and Supplementary Fig. S31). We characterized the successful targeting of our virus in the hippocampus by showing immunofluorescent localization of exogenously transduced Grx1 in the hippocampus of WT and APP/PS1 mice (Fig. 6D) and this was seen only in AAV6-Grx1-injected mice but not in vehicle-injected mice.

\section{Exogenous expression of Grx1 reduces cognitive deficits and restores F-actin levels in APP/PS1 mice}

We then examined if overexpression of Grx 1 could reverse memory deficits measured using the contextual fear condi- tioning (cFC) paradigm. To examine this, we stereotaxically injected either vehicle or AAV6-Grx1 into the hippocampus of 6-month-old WT and APP/PS1 mice. Four weeks after viral transduction, the mice were subjected to $\mathrm{cFC}$ and memory recall was tested $24 \mathrm{~h}$ later in the same setting. $\mathrm{Re}-$ markably, exogenous expression of Grx1 significantly increased freezing response to the context in APP/PS1 mice at $24 \mathrm{~h}$ (Fig. 7A, B) in comparison with vehicle-injected APP/ PS1 mice. However, no comparable effect was observed in either vehicle- or AAV6-Grx1-injected WT mice.

We further studied whether overexpressed Grx1 regulated oxidative stress by measuring ROS levels in hippocampus lysates using $\mathrm{H}_{2}$ DCFDA. As expected, exogenous expression 
A
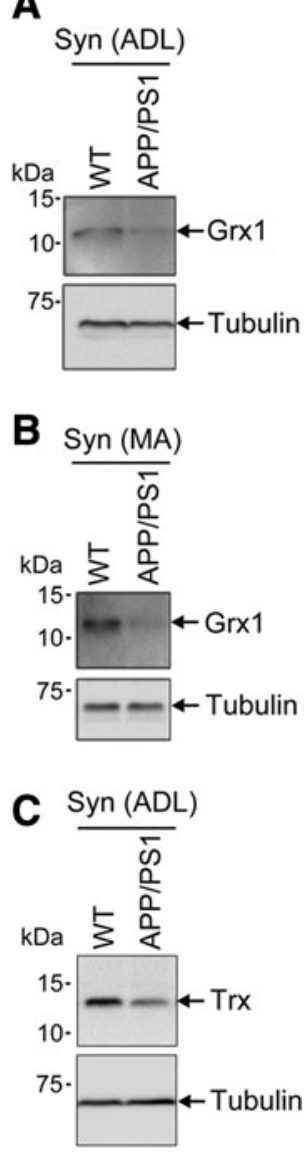

D $\frac{\operatorname{Syn}(\mathrm{MA})}{\ulcorner}$

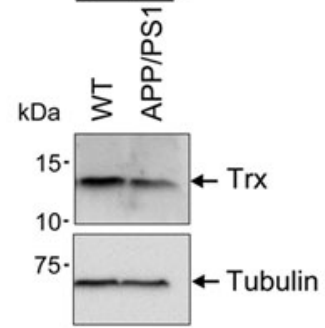

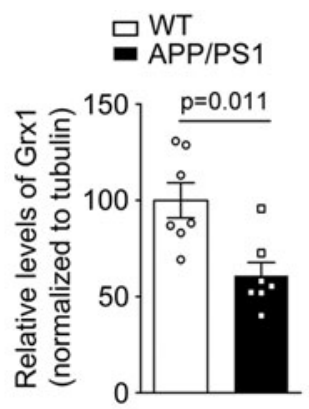
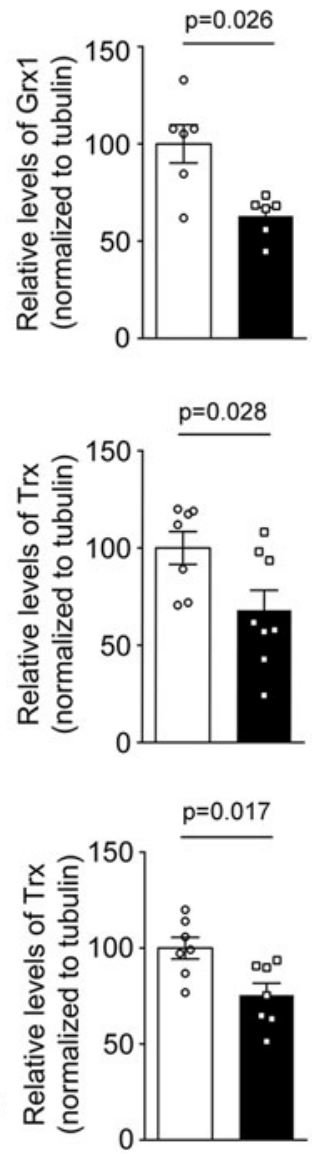

FIG. 5. Grx1 and Trx protein levels are decreased in synaptosomes of APP/PS1 mice. Synaptosomes isolated from ADL and MA WT and APP/PS1 mouse brain cortex samples were subjected to SDS-PAGE and immunoblotted for (A, B) Grx1 and (C, D) Trx. Densitometric analyses for Grx 1 and Trx levels are normalized to tubulin. Statistical analysis: unpaired, two-tailed Mann-Whitney U test. WT versus APP/PS1; " $p$ " values are indicated above the histograms. $p<0.05$ is considered statistical significance between WT and APP/PS1. Results are represented as mean \pm SEM of 6-8 animals per group. Full-length unedited immunoblots for $(\mathbf{A}-\mathbf{D})$ are represented in Supplementary Figures S27-S30. Grx1, glutaredoxin 1; Trx, thioredoxin.

of Grx1 significantly decreased ROS in the hippocampus of APP/PS1 mice (Fig. 7C, D) when compared with vehicleinjected APP/PS1 mice. However, no significant difference was observed in either vehicle- or AAV6-Grx1-injected WT mice.

Furthermore, exogenous expression of Grx1 significantly restored F-actin levels in 6-month-old APP/PS1 mice (Fig. 7E, F, and Supplementary Fig. S32). No such change was seen in hippocampal lysates from 1- (Supplementary Fig. S2) and 6-month-old APP/PS1 mice (Fig. 7GJ; Supplementary Figs. S33 and S34). Collectively, our results demonstrate that synaptosomal actin glutathionylation plays a critical role in F-actin loss and behavioral deficits, and reduction of actin glutathionylation by overexpressing Grx1 restores synaptosomal F-actin as well as memory deficits (cFC). Further, Grx1 overexpression increased levels of postsynaptic density protein 95 (PSD95) but not synaptophysin in lysates from hippocampus (Supplementary Figs. S3, S35, and S36).

Recovery of F-actin loss, in vitro, by overexpression of Grx1 in primary cortical neurons derived from APP/PS1 mice

We then examined if the F-actin loss seen in primary neurons from APP/PS1 mice could be reversed by Grx1 overexpression. At day-in-vitro 18 (DIV18), cortical neurons from APP/PS1 and WT mice were transduced with AAV6-Grx1. After $72 \mathrm{~h}$ of transduction, neuronal cultures were fixed and stained with fluorescently tagged phalloidin, a toxin that binds to most forms of F-actin. Confocal images of at least two segments $(50 \mu \mathrm{m}$ length each) of tertiary neurites from each neuron were captured before automated analysis (MetaMorph). As expected at DIV21, a significant decrease of phalloidin-stained F-actin was observed in tertiary neurites of APP/PS1 primary cortical neurons (Fig. 8A-C). In marked contrast to this, overexpression of Grx1 (AAV6-Grx1) dramatically increased phalloidinstained F-actin in tertiary neurites of APP/PS1 primary cortical neurons (Fig. 8A-C). Our results indicate that prevention of glutathionylation and restoration of F-actin by Grx 1 enhance the polymerization of $\mathrm{G}$-actin to F-actin and prevent $\mathrm{A} \beta$ induced disassembly of F-actin to G-actin.

We performed single-molecule localization microscopy based on direct stochastic optical reconstruction microscopy (dSTORM) on phalloidin-labeled F-actin primary cortical neurons (DIV21) to evaluate the nanoscale organization of polymerized F-actin distribution at excitatory synapses. The organization of F-actin filaments in excitatory synapses was compared between WT, APP/PS1, APP/PS1 neurons infected with AAV6-Grx1, and WT neurons infected with AAV6-Grx1 (Fig. 9A, B, and Supplementary Fig. S4). The presence of Homer1c was considered a positive marker for excitatory synapses (34). An objective classification was used to measure the cumulative distribution of F-actin (40) in excitatory synapses. dSTORM measurements have previously been used to identify the correlative changes in the total content of Factin in the dendritic spines. The differences seen after Grx 1 overexpression were robust and predicted the changes of cumulative F-actin content. Thus, the total F-actin length as observed by a combination of phalloidin staining and single-molecule-based super resolution microscopy is referred as "cumulative length of F-actin." The cumulative length of F-actin was compared between different conditions for synapses that were coimmunostained for Homer 1c (Fig. 9B-H). From the skeletonized distribution of Factin in the postsynaptic compartment, we were able to confirm that the cumulative length of F-actin in spines was significantly decreased in neurons from APP/PS1 mice 


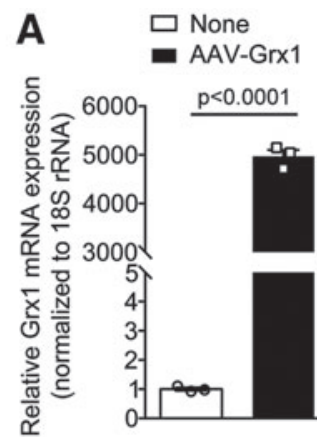

B

Primary cortical neurons

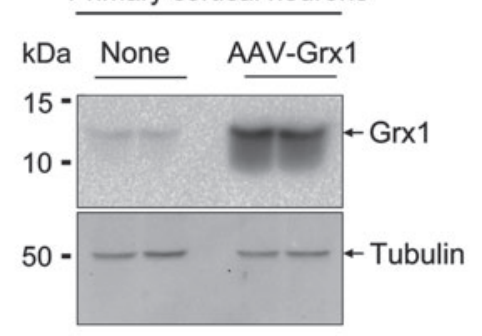

C $\square$ None

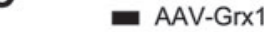

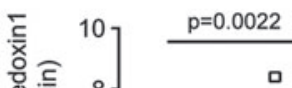

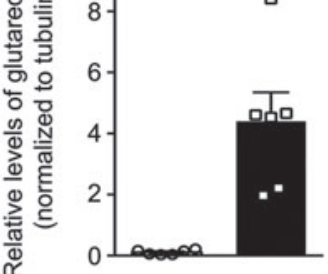

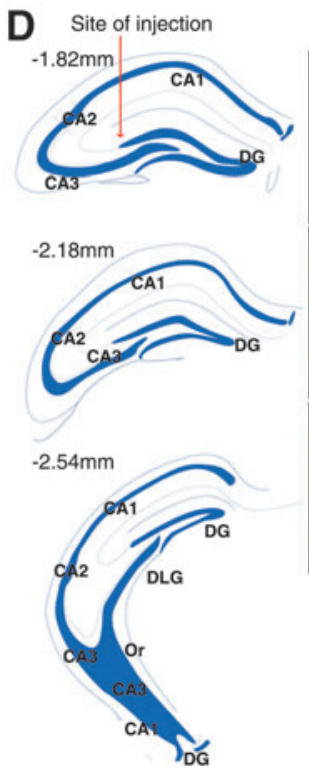

WT+AAV-Grx1 APP/PS1+AAV-Grx1 WT+Vehicle APP/PS1+Vehicle

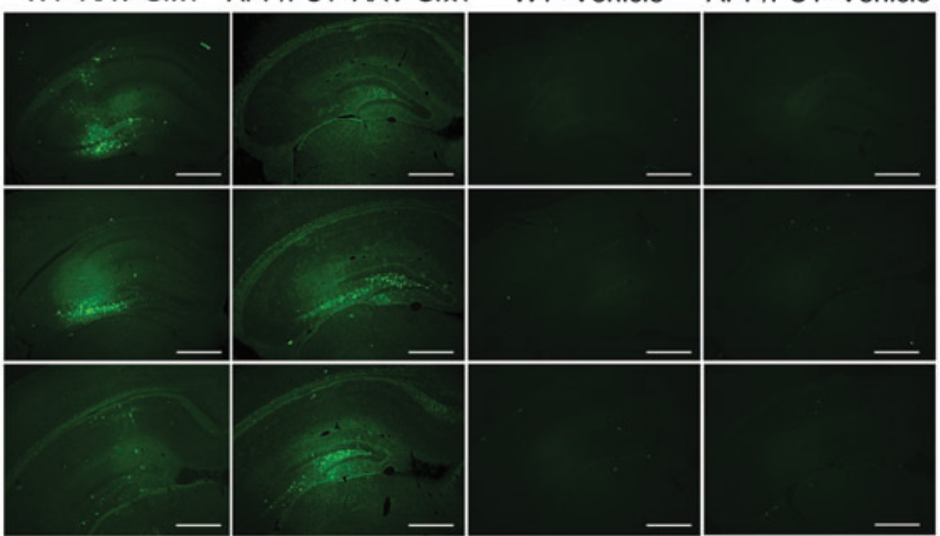

FIG. 6. Biochemical characterization of AAV6-Grx1. AAV6-Grx1 virus was transduced into primary cortical neurons (DIV18) isolated from mixed P0-P1 WT and APP/PS1 mice. At DIV21, transduced neuronal cultures were lysed for RNA and protein analysis. (A) Relative Grx1 mRNA expression was measured by quantitative real-time polymerase chain reaction and graph represents mean \pm SEM from three independent experiments. Unpaired, two-tailed Mann-Whitney U test. (B) Primary cortical neuronal lysates were subjected to SDS-PAGE and immunoblotted for Grx1. (C) Densitometric analyses for Grx1 levels are normalized to tubulin. Graph represents mean \pm SEM from six independent experiments. Unpaired, two-tailed Mann-Whitney $\mathrm{U}$ test. Full-length unedited immunoblots for (B) are represented in Supplementary Figure S31. (D) AAV6-Grx1 (0.2 $\mu$ L of $1.26 \times 10^{11} \mathrm{TU} / \mathrm{mL}$ ) or vehicle was stereotaxically injected bilaterally into the hippocampus of WT and APP/PS1 mouse brain. Four weeks after stereotaxic injections, mice were perfused, and whole brains were removed and fixed in paraformaldehyde. Brains were serially sectioned at $5 \mu \mathrm{m}$ per section. Hippocampal sections were immunostained for Grx 1 antibody. All scale bars are $5 \mu \mathrm{m}$. AAV, adeno-associated virus; DIV, day-in-vitro; TU, transducing unit.

compared with WT. Upon transduction of AAV6-Grx1, the cumulative length of F-actin increased in APP/PS1 neurons and was similar to neurons from WT mice. Furthermore, overexpression of Grx1 has no effects on the cumulative length of F-actin in neurons from WT mice.

\section{Discussion}

Previous work from our laboratory has demonstrated that synaptosomal F-actin levels were decreased concomitantly with increased G-actin in the cerebral cortex of APP/PS1

FIG. 7. Long-term fear memory, F-actin levels are altered by exogenous expression of Grx1 in 6-month-old APP/ PS1 mice. (A) Schematic of the cFC behavioral design. (B) Vehicle or AAV6-Grx1 was stereotaxically injected bilaterally into the hippocampus. Four weeks after stereotaxic injections, WT and APP/PS1 mice were taken for cFC behavioral memory tests and the freezing response was measured. Data represent mean \pm SEM. " $p$ " values are mentioned above the histograms. Two-way ANOVA, Tukey's post hoc test ( $n=8$ per group). (C) Rate of DCF fluorescence was measured from hippocampal lysates. (D) Bar graphs show relative DCF levels in hippocampal lysates. Data represented as mean \pm SEM. Two-way ANOVA, Tukey's post hoc test ( $n=4$ per group). (E-J) F-actin and G-actin fractions isolated from hippocampus lysate samples. These fractions were resolved on stain-free gels and immunoblotted using the monoclonal anti-actin antibody. Relative densitometric analysis for actin levels from F-actin (F), G-actin (H), and lysate (J) fractions was normalized to total lane proteins quantified by fluorescence of TGX stain-free blots. Results are represented as mean \pm SEM. Statistical analysis: two-way ANOVA, Tukey's post hoc test ( $n=6$ per group). Full-length unedited stain-free blots and immunoblots for (E, G, and I) are represented in Supplementary Figures S32-S34. ANOVA, analysis of variance; cFC, contextual fear conditioning. 
A Contextual fear conditioning (cFC)

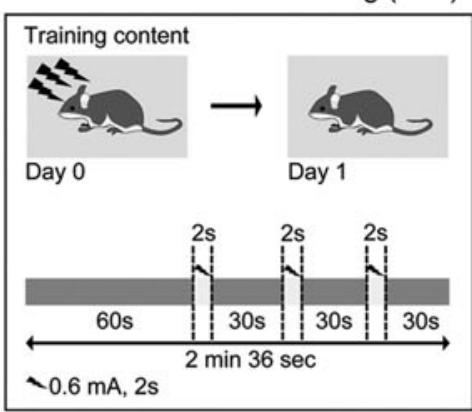

B

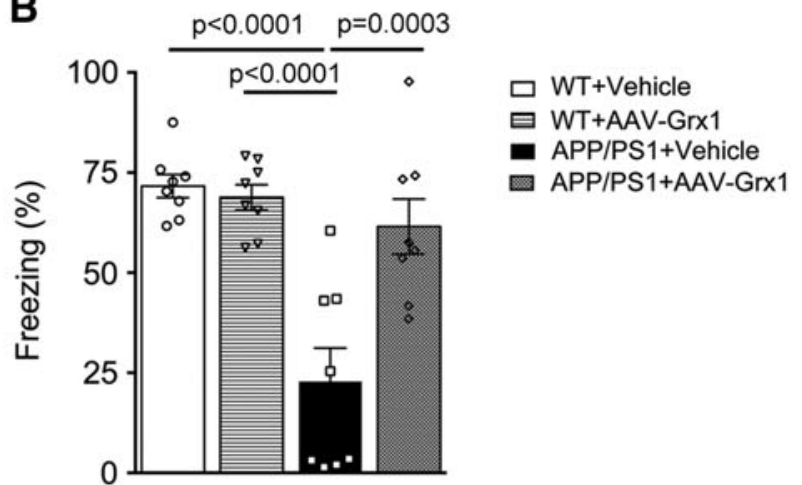

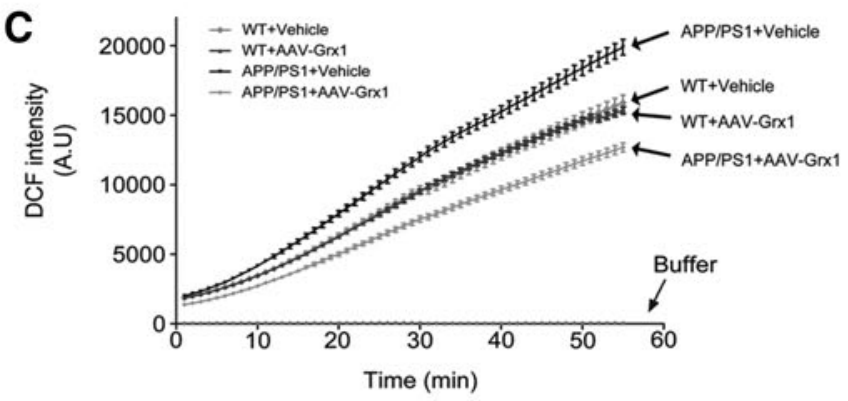

E

F-Actin Fraction

कू कू क

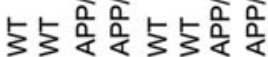

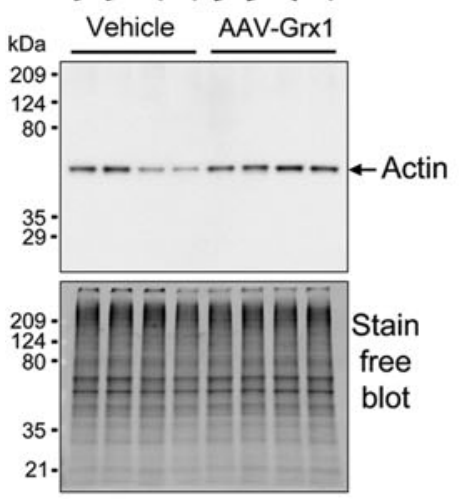

H $\quad$ WT+Vehicle

ㅂTt+AAV-Grx1

- APP/PS1+Vehicle

- APP/PS1+AAV-Grx1

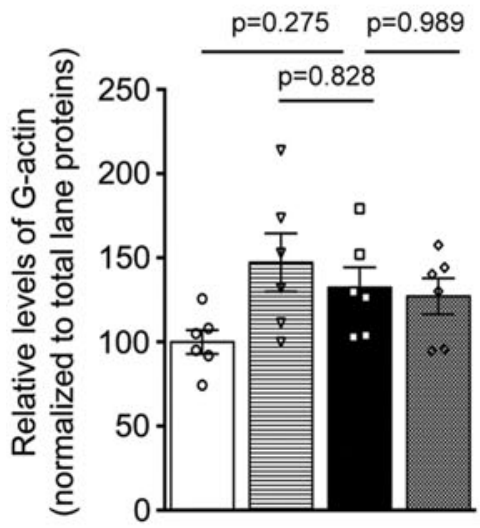

$\mathbf{F}$

ㅁ WT+Vehicle

ㅂ WT+AAV-Grx1

- APP/PS $1+$ Vehicle

- APP/PS1+AAV-Grx

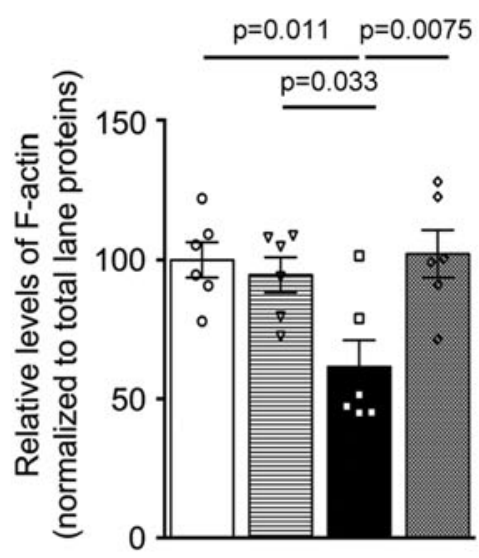

I

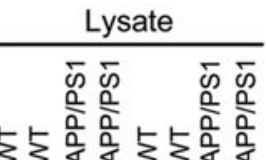

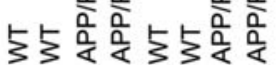

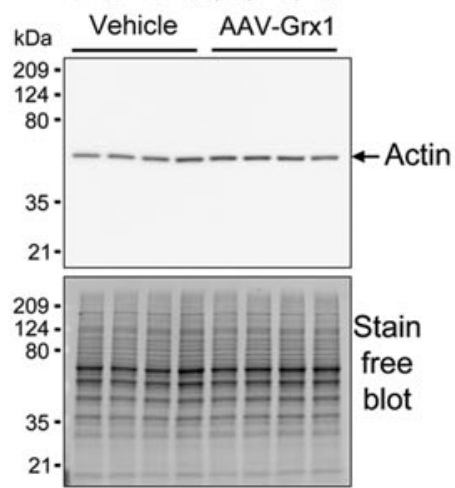

G

G-Actin Fraction

कू कू कू

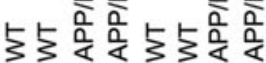

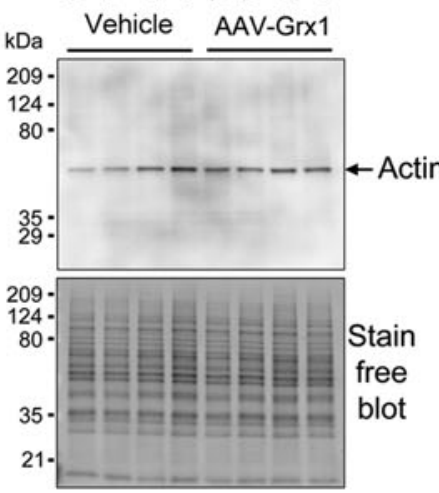

J
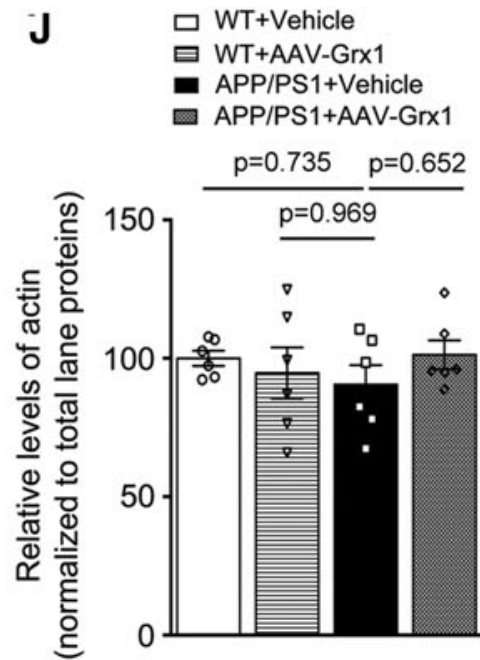
A
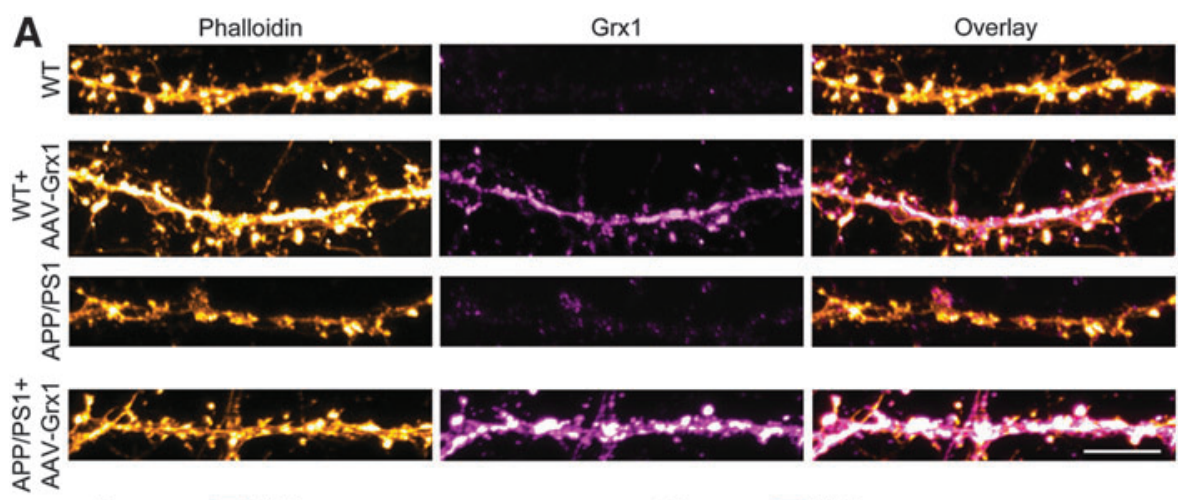
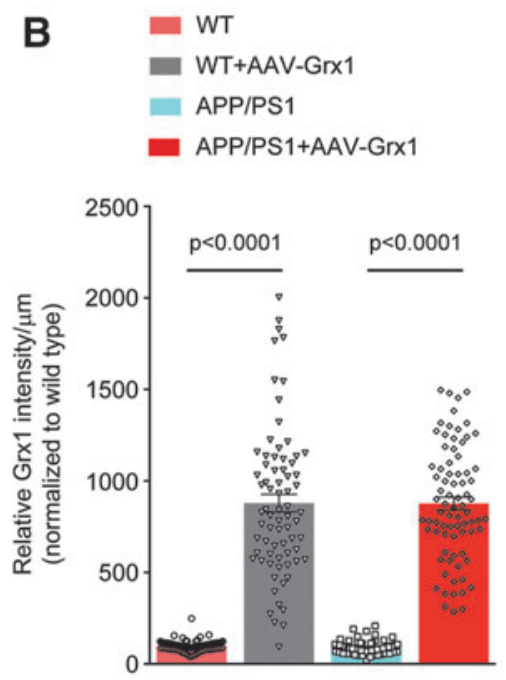

C $=W T$

WT+AAV-Grx1 APP/PS1

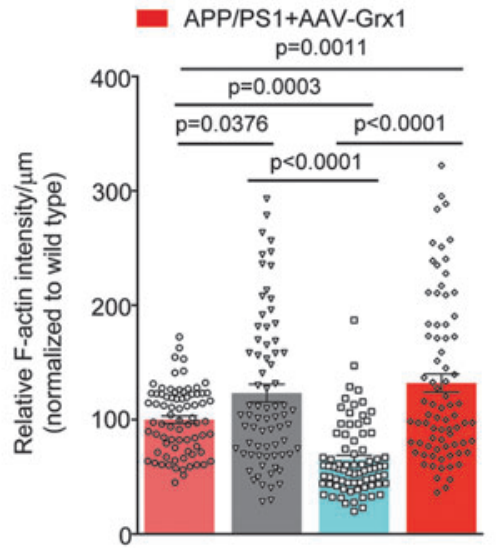

FIG. 8. Exogenous expression of Grx1 in APP/PS1 primary cortical neurons reverses F-actin in tertiary neurites. Representative confocal images of tertiary neurites of primary cortical neurons at DIV21 from WT and APP/PS1 mice littermates. (A) Signals for phalloidin staining and Grx1 immunostaining are shown in orange and magenta, respectively. Scale bar is $5 \mu \mathrm{m}$. (B) Quantification for Grx1 fluorescence intensity; $p<0.0001$, WT versus WT + AAV-Grx1; $p<0.0001$, APP/PS1 versus APP/PS1 + AAV-Grx1, and (C) quantification of F-actin fluorescence (phalloidin) intensity; $p=0.0376$, WT versus WT + AAV-Grx $1 ; p=0.0003$, WT versus APP/PS1; $p<0.0001$, WT + AAV-Grx1 versus APP/PS1; $p<0.0001$, APP/PS1 versus APP/ PS1 + AAV-Grx1; $p=0.0011$, WT versus APP/PS1 + AAV-Grx1. Data are presented as mean \pm SEM from three independent experiments. Statistical analysis for $(\mathbf{B}, \mathbf{C})$ : two-way ANOVA, Tukey's post hoc test. $n=72-78$ neurites $(12,13$ neurons and $24-$ 26 tertiary neurites from each independent experiment).

mice as early as 1 month (34). In this report, we also reproduced our previous results (Fig. $2 \mathrm{G}, \mathrm{H}$ ) that F-actin disassembly and long-term fear memory deficits occur in APP/ PS1 mice early in their life span (34). However, the molecular mechanisms underlying the imbalance of F-actin dynamics in $\mathrm{AD}$ are as yet unclear. Our current results show that the reduced form of F-actin but not G-actin was significantly decreased in synaptosomes of 1- and 9-month-old APP/PS1 mice (Fig. 2A, B, D, and E), indicating that F-actin undergoes oxidative modification. This was observed as glutathionylated actin following immunoprecipitation (IP) of lysates from synaptosomes with antibody to GSH. Furthermore, we found that glutathionylation of F-actin and G-actin was significantly increased in F-actin fractions and G-actin fractions in synaptosomes of APP/PS1 mice. Thus, the glutathionylation seen in G-actin fractions could represent the depolymerization of F-actin. It can be seen that glutathionylation of F-actin is considerably more than that seen on G-actin.

Glutathionylation of the cysteine residue 374 in actin is known to decrease the polymerization rate of actin (68) in A431 and dHL60 cells. Therefore, glutathionylation of F- actin presumably can cause dysregulation of F-actin dynamics. We therefore overexpressed Grx 1 in the mouse brain hippocampus using AAV6-Grx1 and found that the $\mathrm{cFC}$ recall deficit could be reversed significantly (Fig. 7B). Overexpression of Grx 1 in APP/PS1 mice decreased ROS levels (Fig. 7C, D) and restored F-actin levels in the hippocampus (Fig. 7E, F). Although we did not determine if the Cys374 was indeed glutathionylated, nevertheless overexpression of Grx1 was able to restore spine morphology through maintenance of F-actin levels and helped prevent behavioral deficits. This was confirmed using dSTORM, in which Grx1 overexpression restores F-actin nanoassembly in the spine, thus ensuring the preservation of nanoarchitecture of dendritic spines lost in neurons from APP/PS1 mice (Fig. 9). This is very important since dendritic spines are critical for synaptic function, including synaptic plasticity. Our results are in agreement with other studies that have shown the presence of increased S-glutathionylation of specific proteins in AD patients and mouse models $(3,41,56,70)$. Studies with human postmortem AD frontal cortex tissues suggest that oxidative stress is an early feature and plays a critical role in AD 
FIG. 9. Quantification of the nanoarchitecture of $F$ actin in spines of WT and APP/PS1 mice. The four rows of images correspond to spines from primary cortical neuronal cultures from WT, WT plus AAV-Grx1, APP/ PS1, APP/PS1 plus AAVGrx1, respectively (i, vi, xi, xvi); reconstructed dSTORM image of F-actin counterstained with phalloidin Alexa 647 (ii, vii, xii, xvii); rendered dSTORM image of F-actin as a network of ridges (iii, viii, xiii, xviii); dSTORM image of antibody-stained Homer 1c image (iv, ix, xiv, xix); composite of dSTORM image of Homer 1c and rendered dSTORM image of F-actin ( $\mathbf{v}$, $\mathbf{x}, \mathbf{x v}, \mathbf{x x})$; overlay of rendered dSTORM image of F-actin and ridges. (B) F-actin ridges' cumulative length quantification $\mathrm{WT}=5444 \pm 387 \mathrm{~nm}$; WT $+\mathrm{AAV}-\mathrm{Grx} 1=4688 \pm 303 \mathrm{~nm}$; APP/PS1 $=2568 \pm 188 ; \quad$ APP/ PS1 + AAV-Grx1 $=5279 \pm$ $442 \mathrm{~nm} ; n=40(\mathbf{C}-\mathbf{H})$ pairwise comparison between different groups.

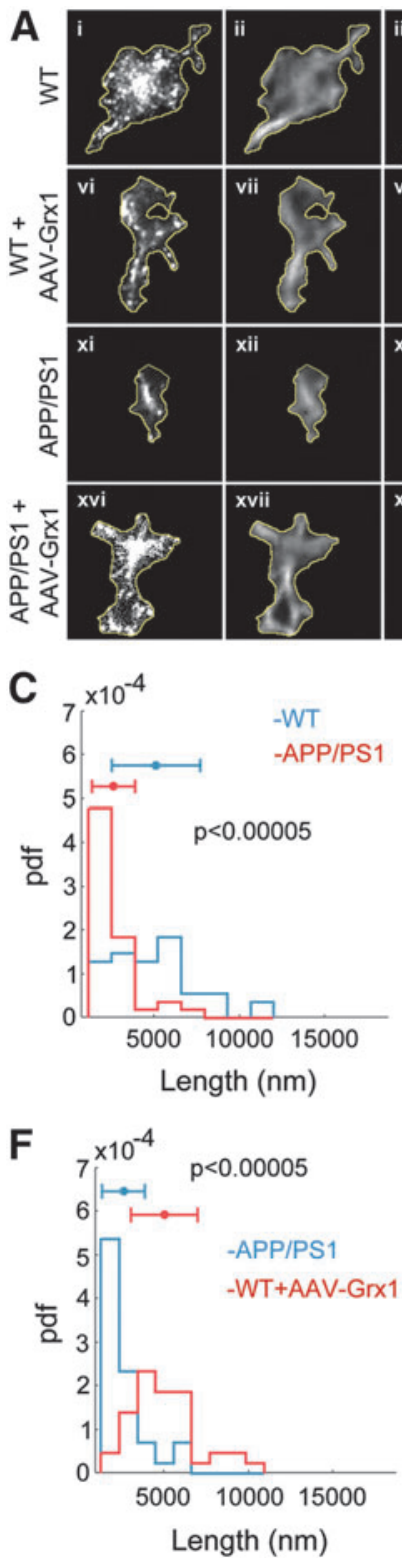

pathogenesis (5). In addition, actin oxidation has also been shown in human postmortem AD superior and middle temporal gyri tissues $(2,11)$. Since we now show that this event occurs very early in $\mathrm{AD}$ pathogenesis and is specifically targeted to the synapse (no change seen in reduced actin levels in the PNS; Fig. 3), it offers an excellent target for early intervention.

Perturbations in PrSH/disulfide homeostasis occur in response to specific oxidative events (38), in a compartmentalized manner, and can modify critical cysteine residues in actin, such as glutathionylation of actin at cysteine-374 (15, 58). Glutathionylated actin inhibits actin polymerization (15). In addition, disruption of Grx 1 results in elevated levels of actin glutathionylation and diminished F-actin dynamics (52). Reactive cysteine residues in proteins are prone to rapid glutathionylation of thiol groups, which acts as a barrier to further oxidation to sulfinic and sulfonic acids, $[\mathrm{R}-(\mathrm{S}=\mathrm{O})-$ $\mathrm{OH}), \mathrm{R}-\mathrm{S}(=\mathrm{O})_{2}-\mathrm{OH}$, which are irreversible. Grx1 is a key enzyme that mediates both glutathionylation and deglutathionylation utilizing equivalent moles of NADPH, and
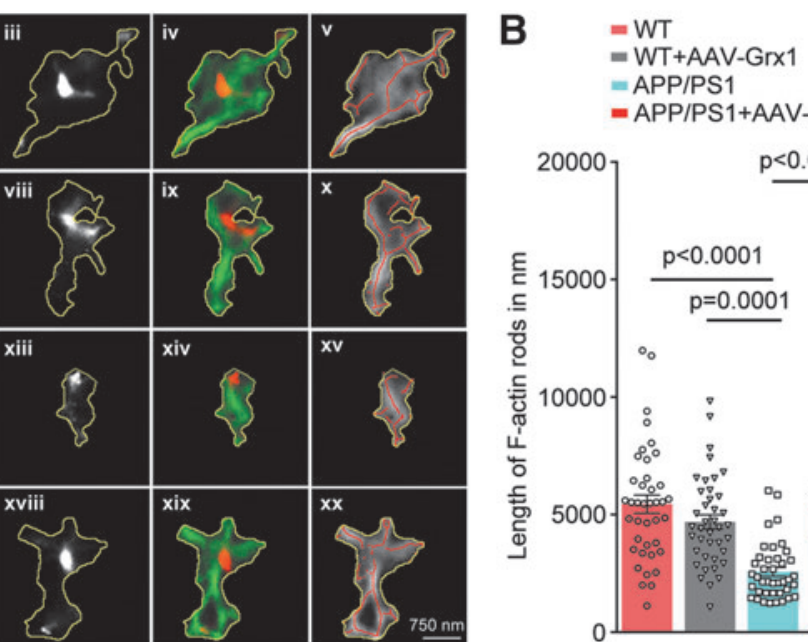

$=$ APP/PS1

- APP/PS1+AAV-Grx1
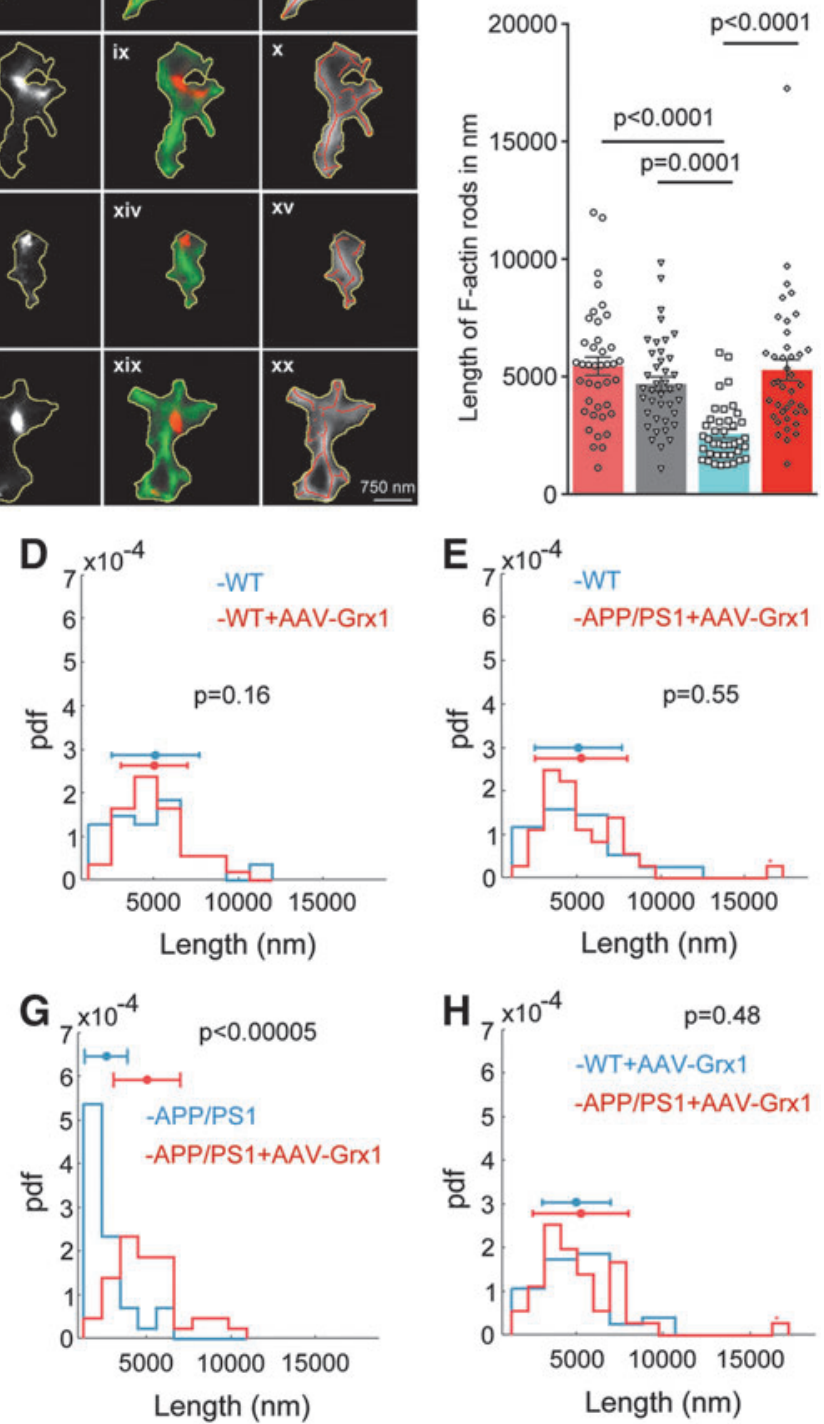

thus plays a very important role in protecting proteins from excessive, irreversible oxidative modifications.

S-glutathionylation of Cys374 in actin is known to shift the ratio of $\mathrm{F}$ - to $\mathrm{G}$-actin, which leads to decreased polymerization of G-actin to F-actin, specifically in the Y-branched networks. It may be noted that in synaptosomes from the cortex of 1month-old APP/PS1 mice, we see decreased F-actin levels concomitant with an increase in G-actin levels (Fig. 2G, H), indicating a similar phenomenon occurring in vivo in mouse brain during AD pathogenesis. This observation, coupled with the results of IP experiments in which increased glutathionylation of actin and decreased Grx1 and Trx levels are seen, suggests that oxidative stress resulting in glutathionylation of actin at the synapse leads to altered actin assembly and disequilibrium of PrSH homeostasis at the synapse in AD brain. In addition to glutathionylation, oxidation of methionine to methionine sulfoxides could also contribute to perturbation of actin cytoskeleton, and also potentially affect interaction between actin and actin binding proteins. Another consequence 
could be the formation of intramolecular disulfide bonds between cysteine-374 and cysteine-285 $(20,21)$ leading to altered actin dynamics. The loss of reduced actin shown in Figure 2 would thus represent contribution from glutathionylation of actin and formation of disulfide bonds. It is needless to mention that oxidative change could also include irreversible modifications, such as tyrosine nitrosylation. We did look for but were unable to detect carbonylation of proteins in synaptosomes from 1-month-old APP/PS1 mice.

Grx1 is the principal thiol/disulfide oxidoreductase (thioltransferase) in the cell, restoring protein-SH and protein function by deglutathionylation [(29); Fig. 4D]. Studies have shown that Grx1 protects neuronal degeneration both in vitro and in vivo $(33,46,49,51)$. Grx1 is a positive physiological regulator of actin polymerization (28). Here we show that synaptosomal Grx1 and Trx levels were significantly decreased in 1-month-old APP/PS1 mice (Fig. 5) and this decrease was sustained up to 9 months of age (when overt pathological hallmarks are seen), indicating that synaptosomal Grx1 and Trx levels are decreased through the observed life span of the APP/PS1 mice. Lower levels of Grx 1 are known to result in decreased capacity to reduce PrSSG to thiols, which may be highly compromised in the synapse of APP/PS1 mice.

Grx1 plays a critical role in maintaining thiol/disulfide homeostasis, particularly in terms of the F-actin cytoskeleton in dendritic spines. Therapeutic strategies focused on enhancing the expression of Grx1 early in the disease process could help negate F-actin disassembly and thus help maintain synaptic plasticity and improve memory recall. Several transcription factor binding sites have been identified in the human Grx1 promoter (namely, AP1, AREB6, ATF2, and cJun). Small molecules targeted to augment the activation of these transcription factors could potentially facilitate enhanced expression of Grx1 in the brain and help restore synaptic plasticity during AD pathogenesis.

\section{Materials and Methods}

\section{Reagents}

Deoxyribonuclease I (Cat. No. D-4513), papain (Cat. No. P4762), and poly-D-lysine hydrobromide (Cat. No. P6407) were obtained from Sigma-Aldrich. AMS (Cat. No. A485), $\mathrm{H}_{2}$ DCFDA (Cat. No. D399), DCF (Cat. No. N7991), Dynabeads Protein G (Cat. No. 10003D), ProLong Gold Antifade Mountant with 4',6-diamidino-2-phenylindole (DAPI, Cat. No. P36931), GlutaMAX (Cat. No. 35050061), penicillin/streptomycin (Cat. No. 15140122), Neurobasal-A medium (Cat. No. 10888022), B27 Supplement (Cat. No. 175040444), and Pierce bicinchoninic acid (BCA) protein assay kit were purchased from Thermo Fisher Scientific, Inc. (Waltham, MA). The G-Actin/F-actin In Vivo Assay Biochem Kit (Cat. No. BK037) was obtained from Cytoskeleton, Inc. (Denver, CO). TGX Stain Free Fast Cast Acrylamide kits (Cat. No. 1610185), Clarity western-enhanced chemiluminescent substrate (Cat. No. 1705061), and ImmunBlot polyvinylidene difluoride membrane (Cat. No. 1620177) were purchased from Bio-Rad Laboratories, Inc. All other chemicals were obtained from Sigma-Aldrich (St. Louis, MO).

\section{Antibodies}

Acti-Stain 488 (Cat. No. PHDG1) was purchased from Cytoskeleton, Inc. Mouse monoclonal antibody against $\beta$ - actin was obtained from MP Biomedicals (Cat. No. 691002; Santa Ana, CA). Anti- $\beta$-tubulin monoclonal antibody (Cat. T8328; Sigma), rabbit polyclonal antibody against $\beta$-actin (Cat. No. ab16039; RRID:AB_956497), anti-Grx1 (Cat. No. ab45953; RRID:AB_880242), anti-Trx (Cat. No. ab86255; RRID:AB_1925501), anti-synaptophysin (Cat. No. ab14692; RRID:AB_301417), anti-PSD95 (Cat. No. ab18258; RRID:AB_444362), and anti-SNAP29 (Cat. No. ab138500; RRID:AB_2687667) were purchased from Abcam. Anti- $\alpha-$ synuclein (Cat. No. 4179; Cell Signaling Technology, Inc.), anti-GSH monoclonal antibody (Cat No. 101-A-100; Virogen), and Homer1c (Cat No. 160003, RRID:AB_887730; Synaptic Systems, Germany) were purchased. Horseradish peroxidase-conjugated secondary antibodies and normal goat serum blocking solution (Cat. No. S-1000) were purchased from Vector Laboratories, Inc. (Burlingame, CA). Alexa Fluor 647 phalloidin (Cat. No. A22287) was obtained from Thermo Fisher Scientific, Inc.

\section{Experimental mice}

$\mathrm{APP}_{\mathrm{Swe}} / \mathrm{PS} 1 \Delta \mathrm{E} 9$ double transgenic mice were procured from Jackson Laboratory. WT and APPswe/PS1 $\Delta E 9$ (APP/ PS1) mice were bred at the Institutional Central Animal Facility and 1-month-old (30-35 days; ADL), 6-month-old (180-190 days), and 9-month-old (270-300 days; MA) male mice were used for the experiments. Mice were housed and maintained under pathogen-free conditions in a temperaturecontrolled room on 12-h light/12-h dark cycle and had ad libitum access to food and water (34). All animal experiments were carried out in accordance with Institutional Guidelines for the Care and Use of Laboratory Animals under approval of the Institutional Animal Ethics Committee.

\section{Contextual fear conditioning}

Behavioral experiments were carried out with 6-monthold male mice as described (34). Before cFC experiments, mice were housed individually and were handled for $5 \mathrm{~min}$ for three consecutive days. Briefly, mice were allowed to explore the training context for 1 min during training day and then received three foot shocks, $(2 \mathrm{~s}$ and $0.6 \mathrm{~mA}$ each, intertrial interval: $30 \mathrm{~s}$ ). We assessed cFC memory by returning mice to the training context $24 \mathrm{~h}$ after training and measuring the freezing during a test period of $2 \mathrm{~min}$. Freezing was defined as complete absence of somatic mobility other than respiratory movements. No animals were excluded from the analysis.

\section{Stereotaxic surgery}

Mice were weighed and anesthetized with ketamine/xylazine mixture and surgeries were conducted under aseptic conditions using a small-animal stereotaxic instrument (Dual lab standard stereotaxic module Stoelting Co., Wood Dale, IL), and body temperature was maintained with a heating pad until the completion of surgical procedure and recovery from anesthesia. Vehicle or AAV6-Grx1 virus was delivered bilaterally into the hippocampus of WT and APP/PS1 mice using a $22 \mathrm{G}$ blunt tip Hamilton needle and syringe (Hamilton Co., Reno, NV). Stereotaxic injection coordinates relative to bregma are as follows: anteroposterior -2.40 , mediolateral \pm 2.00 , dorsoventral (relative to dura) -2.30 . The needle was 
inserted at the desired coordinates and $\sim 200 \mathrm{~nL}$ of the vehicle or virus preparation was slowly injected over a period of 2 min. After the end of the injection, the needle was allowed to remain in position for a further $10 \mathrm{~min}$ to allow virus diffusion before retraction. The injection site is depicted in a cartoon in Figure 6D. Mice were observed daily to monitor their health after surgical recovery. To allow for transgene expression, mice were kept under home cage conditions for 4 weeks before any behavioral tests and biochemical experiments.

\section{Synaptosomal preparation}

Synaptosomes were prepared as described (1). Mice were anesthetized and decapitated. The brain cortex was dissected out and homogenized. The homogenate was centrifuged at $1500 \mathrm{~g}$ for $10 \mathrm{~min}$ at $4^{\circ} \mathrm{C}$. The PNS was further centrifuged at $12,000 \mathrm{~g}$ for $15 \mathrm{~min}$ at $4^{\circ} \mathrm{C}$, and the pellet was resuspended. The resuspended pellet was then layered over a discontinuous sucrose gradient (0.85-1.0-1.2 $M$ ) and centrifuged at $85,000 \mathrm{~g}$ for $2 \mathrm{~h}$ at $4^{\circ} \mathrm{C}$. Synaptosomal fraction obtained at the interface of 1 and 1.2 $M$ sucrose was collected, washed twice in $5 \mathrm{~m} M$ Tris $\mathrm{pH} \mathrm{8.1,} \mathrm{and} \mathrm{resuspended} \mathrm{in} \mathrm{homogeni-}$ zation buffer.

\section{Preparation of G-actin and F-actin fractions}

G-actin and F-actin fractions were prepared from synaptosomes and PNS as described (34). Protein concentrations were determined using the BCA protein assay kit before immunoblotting. All fractions were resolved using the TGX Stain Free Fast Cast Acrylamide kit, 12\% or sodium dodecyl sulfate/polyacrylamide gel electrophoresis (SDS-PAGE) gels, and the standard immunoblotting protocol was followed. Stain-free blots were imaged before antibody incubations using Chemidoc-XRS (Bio-Rad) and analyzed with Image lab software. Immunoreactive bands were detected using enhanced chemiluminescence, and images were acquired using Chemidoc-XRS and analyzed.

\section{AMS derivatization of thiols}

PNS, synaptosomes, and G-actin and F-actin fractions were chemically labeled with thiol alkylating agent, AMS (15 $\mathrm{mM})$, in a denaturing buffer, as described previously (44). Excess AMS was quenched with reduced GSH $(30 \mathrm{mM})$ and derivatized samples were resolved under reducing conditions, and the standard immunoblotting procedure was followed. $\alpha$-Synuclein or SNAP29 was used as loading controls for $\mathrm{G}$-actin and $\mathrm{F}$-actin fractions.

\section{Detection of actin S-glutathionylation by IP}

In brief, brain cortex synaptosomes, and F-actin and Gactin fractions isolated from synaptosomes were resuspended in an ice-cold Nonidet P-40 lysis buffer containing phosphatase and protease inhibitors and incubated with the antiGSH antibody $(2 \mu \mathrm{g} / \mathrm{mL})$ at $4^{\circ} \mathrm{C}$ with end-over-end rotation overnight, and then $30 \mu \mathrm{L}$ of Dynabeads Protein $\mathrm{G}$ was added to the solution and incubated for $4 \mathrm{~h}$ at $4^{\circ} \mathrm{C}$ on a rocker platform. Immunocomplexes were extensively washed with Nonidet P-40 lysis buffer to remove nonspecific binding. After the final wash, the pellet was resuspended in $2 \times$ SDS-PAGE sample buffer and boiled for $5 \mathrm{~min}$. The im- munoprecipitated proteins were subjected to SDS-PAGE and immunoblotted for anti-actin.

\section{Immunohistochemistry in mouse brain}

Mouse brain (vehicle or AAV6-Grx1 injected) was fixed in $4 \%(\mathrm{w} / \mathrm{v})$ buffered paraformaldehyde. The tissue was processed for paraffin embedding, and serial sections ( $5 \mu \mathrm{m}$ thick) were cut using microtome (Leica Biosystems, Inc., Buffalo Grove, IL). Sections were dewaxed by xylene treatment I and II for $5 \mathrm{~min}$ each followed by rehydration by alcohol series $(100 \%, 90 \%, 70 \%, 50 \%, 30 \%)$ for $2 \mathrm{~min}$. Sections were then given brief washings with water and phosphate-buffered saline (PBS). The sections were pressure cooked for $10 \mathrm{~min}$ in sodium citrate buffer ( $\mathrm{pH}$ 6.8). After cooling, the sections were washed with PBS having Triton X-100 (10 mM, pH 7.4, 0.025\% Triton $\mathrm{X}-100$ ) and incubated in $10 \%$ normal goat serum and $1 \%$ bovine serum albumin (BSA) for $2 \mathrm{~h}$ at room temperature to block nonspecific binding. Next, the sections were incubated with antibody against Grx 1 (1:1000 dilution) overnight at $4^{\circ} \mathrm{C}$. After washing with PBS containing Triton X-100, the sections were incubated in secondary antibody conjugated to AlexaFluor488. Subsequently, the sections were washed and mounted with Vectashield mounting medium without DAPI (H-1000). Image acquisition was performed using $5 \times / 0.13$ air numerical aperture, objective N-Achroplan object, Zeiss Axio Scope.A1 (Carl Zeiss Microscopy, LLC, Thornwood, NY).

\section{Measurement of ROS using $\mathrm{H}_{2}$ DCFDA assay}

The cell permeant, $\mathrm{H}_{2}$ DCFDA, an oxidant-sensitive dye that is converted to DCF by oxidation and after deesterification, was used to measure ROS levels as described previously (1). Briefly, PNS, synaptosomes, or hippocampal tissue lysates were incubated with $10 \mu M \mathrm{H}_{2}$ DCFDA in $0.1 M$ phosphate buffer ( $\mathrm{pH}$ 7.4) for $5 \mathrm{~min}$ at room temperature. Fluorescence was measured by microplate reader (Infinite M200 Pro plate reader; Tecan Group Ltd., Switzerland) using excitation at $488 \mathrm{~nm}$ and emission of $525 \mathrm{~nm}$ every $30 \mathrm{~s}$ for 90 min. A standard curve of DCF was used for quantification of ROS levels.

\section{Primary cortical neuronal culture}

Primary cortical neurons were prepared from postnatal day 0 to postnatal day 1 (P0-P1) WT and APP/PS1 mouse brain cortex. After removing meninges, neurons were dissociated using mechanical trituration in $0.25 \%$ papain $(10 \mathrm{U} / \mathrm{mg})$ solution containing $(1 \times$ Hank's balanced salt solution, $12.5 \mathrm{mM}$ 2-[4-(2-hydroxyethyl)piperazin-1-yl]ethanesulfonic acid, D-glucose, and $500 \mu \mathrm{g} / \mathrm{mL}$ penicillin/streptomycin. Cortical neurons were seeded on coverslips precoated with poly-D-lysine $(0.1 \mathrm{mg} / \mathrm{mL})$ in 12 -well plates. Neurobasal medium supplemented with $0.5 \times$ B27, $2 \mathrm{~m} M$ L-GlutaMAX, and $100 \mu \mathrm{g} / \mathrm{mL}$ penicillin/streptomycin was used to grow the neurons in serum-free medium conditions and maintained at $37^{\circ} \mathrm{C}$ in $5 \% \mathrm{CO}_{2}$ for 3 weeks.

\section{AAV6-Grx1 vector production}

cDNA coding for human Grx1 sequence was subcloned from a pCMV vector backbone into the multiple cloning site of pAAV-MCS (Stratagene, La Jolla, CA) to generate pAAV-Grx1. This construct expresses human Grx1 under the control of CMV promoter with a $\beta$-globin intronic enhancer 
and is flanked by AAV2 inverted repeats. pAAV-CMV-Grx1 was packaged into rAAV serotype 6 (AAV6) particles by cotransfecting the vector into the AAV-293 cell line along with pDF6 plasmid (23) using the calcium phosphate method (63). After $48 \mathrm{~h}$ of transfection, cells were lysed using multiple alternating rounds of freezing in a dry ice/methanol mixture and thawing in a $37^{\circ} \mathrm{C}$ water bath. The lysate was purified and concentrated using high-pressure liquid chromatography through a heparin affinity column as described earlier (64).

\section{AAV6-Grx1 titration}

Infectious particle or viral transducing unit counts were determined using a quantitative PCR method by comparing against a reference AAV6-GFP viral preparation. The absolute TUs for the AAV6-GFP virus were estimated using fluorescence-activated cell sorting analysis as described (63). For quantitative polymerase chain reaction (qPCR)-based estimation, purified AAV6-Grx1 or AAV6-GFP was added in varying serial dilutions onto HEK293T cells. After $48 \mathrm{~h}$ of transduction, cells were harvested and dsDNA containing transduced AAV2 and host cell genomic DNA were purified. qPCR was performed to estimate the copies of $\beta$-globin intronic sequence (present in both the AAV and host cell genomes) and normalized to the copies of albumin (only present in host cell genomes). This normalized value was compared against the reference AAV6-GFP virus to estimate TUs/mL $(47,64)$.

\section{AAV6-Grx1 transduction and staining of primary cortical neurons}

Primary cortical neurons were transduced on DIV18 with AAV6-Grx1 (40 TUs/cell) and experiments were performed on DIV21. Untransduced neurons from the same batch were used as controls. After 3 days of transduction, neurons were washed with ice-cold PBS and fixed for immunostaining or lysed in an ice-cold lysis buffer containing phosphatase and protease inhibitors. The neuronal lysate was centrifuged at $14,000 \mathrm{~g}$ for $30 \mathrm{~min}$ at $4^{\circ} \mathrm{C}$. After centrifugation, protein concentrations were determined by BCA protein assay and subjected to SDS-PAGE followed by immunoblotting.

Primary cortical neurons, untransduced and transduced with AAV6-Grx1, were fixed at DIV21 with 2\% paraformaldehyde for $10 \mathrm{~min}$ at room temperature. Quenching was performed with $0.1 \mathrm{M}$ glycine for $5 \mathrm{~min}$ at room temperature. F-actin was stained with acti-stain 488 phalloidin as per the instructions of the manufacturer. Anti-Grx1 antibody was used at a dilution of 1:1000 for $1 \mathrm{~h}$ at room temperature.

\section{Confocal image acquisition and analysis}

Confocal images were acquired using Carl Zeiss LSM780 using Argon 488 laser for acti-stain 488 phalloidin. Helium/ neon 594 laser was used to visualize Grx1. Oil immersion objective $63 \times / 1.40$ was used, and $z$-stack images were captured using the following parameters: $512 \times 512$ resolution and 12-bit depth, zoom factor of 3 , pinhole 1 airy unit, and step size interval of $0.4 \mu \mathrm{m}$. All confocal images for phalloidin-labeled neurites were acquired under identical conditions and analyzed after blinding.

Quantification of F-actin levels measured as phalloidin intensity was performed using MetaMorph software (Meta-
Morph, version 7.8.0.0, 2013; Molecular Devices, LLC). Confocal z-stack images were loaded onto MetaMorph software and maximum intensity projection was generated. After background subtraction and thresholding, mask was generated around the dendrite of interest (including the spines) and phalloidin intensity was measured.

\section{Immunocytochemistry for dSTORM imaging}

For dSTORM imaging, samples were fixed with $0.3 \%$ glutaraldehyde (v/v) and blocked with $3 \%$ BSA (w/v) containing $0.2 \%$ Triton X-100 (v/v) for $30 \mathrm{~min}$. Homer $1 \mathrm{c}$ antibody was used for immunostaining followed by the Alexa 532 secondary antibody. F-actin labeling was then performed with Alexa 647-Phalloidin $(34,69)$.

\section{dSTORM imaging}

dSTORM imaging of F-actin and Homer1c from primary cortical neurons (DIV21) was done as reported previously (34).

\section{Image analysis}

To objectively analyze dSTORM data sets of F-actin in spines, we performed segmentation followed by automatic ridge detection within spines (40). Using a fully automated and an objective workflow for analysis, we measured the extent of F-actin ridges in excitatory synapses marked positive for Homer1c. The workflow for analysis comprised the following: (i) segmentation to identify Homer-positive spines, (ii) followed by automatic detection of ridges of Factin within spines using Ridge Detection plugin in Image J (57). Briefly, we trained a deep neural network from artificial neural network accelerated-photoactivation localization microscopy (ANNA-PALM) (42) on dSTORM data set of F-actin in neurons and derived a model where F-actin distribution within spines becomes more homogeneous. Images obtained as an output of this model were subsequently used for further segmentation for spine identification. In the next step, we modeled ridges seen in spines with microtubulemodel generated with ANNA-PALM (42). This resulted in a smoothened image of an otherwise pointillistic dSTORM image. We then used upon the Ridge Detection plug-in from Image J to automatically identify the ridges.

\section{Statistical analyses}

Statistical analyses were performed using Prism 7 (GraphPad Prism software). Statistical comparisons between two groups were performed with the two-tailed unpaired Mann-Whitney U test. Two-way analysis of variance with Tukey's post hoc test was used for experiments with four groups. Results are represented as mean \pm standard error of the mean. " $p$ ", values $<0.05$ were considered significant.

\section{Acknowledgments}

The authors are grateful to Prof. Patrick Aebischer (Ecole Polytechnique Fédérale de Lausanne, Switzerland) for providing laboratory resources and the Indo-Swiss Bilateral Research Initiative for providing funding support to A.R. for carrying out AAV production and titration. 


\section{Author Disclosure Statement}

No competing financial interests exist.

\section{Funding Information}

This work was supported by Tata Trusts (V.R.). R.P.K. and D.N. are Ramalingaswami Fellows of the Department of Biotechnology (BT/RLF/Re-entry/50/2014 and BT/RLF/ Re-entry/24/2012). S.N. is supported by the Science and Engineering Research Board (postdoctoral Fellowship PDF/ 2016/003226).

\section{Supplementary Material}

Supplementary Figure S1

Supplementary Figure S2

Supplementary Figure S3

Supplementary Figure S4

Supplementary Figure S5

Supplementary Figure S6

Supplementary Figure S7

Supplementary Figure S8

Supplementary Figure S9

Supplementary Figure S10

Supplementary Figure S11

Supplementary Figure S12

Supplementary Figure S13

Supplementary Figure S14

Supplementary Figure S15

Supplementary Figure S16

Supplementary Figure S17

Supplementary Figure S18

Supplementary Figure S19

Supplementary Figure S20

Supplementary Figure S21

Supplementary Figure S22

Supplementary Figure S23

Supplementary Figure S24

Supplementary Figure S25

Supplementary Figure S26

Supplementary Figure S27

Supplementary Figure S28

Supplementary Figure S29

Supplementary Figure S30

Supplementary Figure S31

Supplementary Figure S32

Supplementary Figure S33

Supplementary Figure S34

Supplementary Figure S35

Supplementary Figure S36

Supplementary Figure S37

\section{References}

1. Ahmad F, Singh K, Das D, Gowaikar R, Shaw E, Ramachandran A, Rupanagudi KV, Kommaddi RP, Bennett DA, and Ravindranath V. Reactive oxygen species-mediated loss of synaptic Akt1 signaling leads to deficient activitydependent protein translation early in Alzheimer's disease. Antioxid Redox Signal 27: 1269-1280, 2017.

2. Aksenov MY, Aksenova MV, Butterfield DA, Geddes JW, and Markesbery WR. Protein oxidation in the brain in Alzheimer's disease. Neuroscience 103: 373-383, 2001.
3. Aksenov MY and Markesbery WR. Changes in thiol content and expression of glutathione redox system genes in the hippocampus and cerebellum in Alzheimer's disease. Neurosci Lett 302: 141-145, 2001.

4. Akterin S, Cowburn RF, Miranda-Vizuete A, Jiménez A, Bogdanovic N, Winblad B, and Cedazo-Minguez A. Involvement of glutaredoxin-1 and thioredoxin-1 in betaamyloid toxicity and Alzheimer's disease. Cell Death Differ 13: 1454-1465, 2006.

5. Ansari MA and Scheff SW. Oxidative stress in the progression of Alzheimer disease in the frontal cortex. $J$ Neuropathol Exp Neurol 69: 155-167, 2010.

6. Arodin L, Lamparter H, Karlsson H, Nennesmo I, Björnstedt M, Schröder J, and Fernandes AP. Alteration of thioredoxin and glutaredoxin in the progression of Alzheimer's disease. J Alzheimers Dis 39: 787-797, 2014.

7. Bamburg JR and Bernstein BW. Actin dynamics and cofilin-actin rods in Alzheimer disease. Cytoskeleton (Hoboken) 73: 477-497, 2016.

8. Bertling E, Englund J, Minkeviciene R, Koskinen M, Segerstråle M, Castrén E, Taira T, and Hotulainen P. Actin tyrosine-53-phosphorylation in neuronal maturation and synaptic plasticity. J Neurosci 36: 5299-5313, 2016.

9. Butterfield DA and Boyd-Kimball D. Oxidative stress, amyloid- $\beta$ peptide, and altered key molecular pathways in the pathogenesis and progression of Alzheimer's disease. $J$ Alzheimers Dis 62: 1345-1367, 2018.

10. Butterfield DA, Di Domenico F, and Barone E. Elevated risk of type 2 diabetes for development of Alzheimer disease: a key role for oxidative stress in brain. Biochim Biophys Acta 1842: 1693-1706, 2014.

11. Butterfield DA, Di Domenico F, Swomley AM, Head E, and Perluigi M. Redox proteomics analysis to decipher the neurobiology of Alzheimer-like neurodegeneration: overlaps in Down's syndrome and Alzheimer's disease brain. Biochem J 463: 177-189, 2014.

12. Butterfield DA and Lauderback CM. Lipid peroxidation and protein oxidation in Alzheimer's disease brain: potential causes and consequences involving amyloid betapeptide-associated free radical oxidative stress. Free Radic Biol Med 32: 1050-1060, 2002.

13. Chazeau A, Mehidi A, Nair D, Gautier JJ, Leduc C, Chamma I, Kage F, Kechkar A, Thoumine O, Rottner K, Choquet D, Gautreau A, Sibarita J-B, and Giannone G. Nanoscale segregation of actin nucleation and elongation factors determines dendritic spine protrusion. EMBO J 33: 2745-2764, 2014.

14. Cheignon C, Tomas M, Bonnefont-Rousselot D, Faller P, Hureau C, and Collin F. Oxidative stress and the amyloid beta peptide in Alzheimer's disease. Redox Biol 14: 450464, 2018.

15. Dalle-Donne I, Giustarini D, Rossi R, Colombo R, and Milzani A. Reversible S-glutathionylation of Cys 374 regulates actin filament formation by inducing structural changes in the actin molecule. Free Radic Biol Med 34: 23-32, 2003.

16. Di Domenico F, Barone E, Perluigi M, and Butterfield DA. The triangle of death in Alzheimer's disease brain: the aberrant cross-talk among energy metabolism, mammalian target of rapamycin signaling, and protein homeostasis revealed by redox proteomics. Antioxid Redox Signal 26: 364-387, 2017.

17. Domenico F Di, Cenini G, Sultana R, Perluigi M, Uberti D, Memo M, and Butterfield DA. Glutathionylation of the proapoptotic protein p53 in Alzheimer's disease brain: impli- 
cations for AD pathogenesis. Neurochem Res 34: 727-733, 2009.

18. Efimova N, Korobova F, Stankewich MC, Moberly AH, Stolz DB, Wang J, Kashina A, Ma M, and Svitkina T. $\beta$ III spectrin is necessary for formation of the constricted neck of dendritic spines and regulation of synaptic activity in neurons. J Neurosci 37: 6442-6459, 2017.

19. Farah ME and Amberg DC. Conserved actin cysteine residues are oxidative stress sensors that can regulate cell death in yeast. Mol Biol Cell 18: 1359-1365, 2007.

20. Farah ME, Sirotkin V, Haarer B, Kakhniashvili D, and Amberg DC. Diverse protective roles of the actin cytoskeleton during oxidative stress. Cytoskeleton 68: 340-354, 2011.

21. Fedorova M, Kuleva N, and Hoffmann R. Reversible and irreversible modifications of skeletal muscle proteins in a rat model of acute oxidative stress. Biochim Biophys Acta 1792: 1185-1193, 2009.

22. García-Ortiz A, Martín-Cofreces NB, Ibiza S, Ortega Á, Izquierdo-Álvarez A, Trullo A, Victor VM, Calvo E, Sot B, Martínez-Ruiz A, Vázquez J, Sánchez-Madrid F, and Serrador JM. eNOS S-nitrosylates $\beta$-actin on Cys374 and regulates $\mathrm{PKC}-\theta$ at the immune synapse by impairing actin binding to profilin-1. PLoS Biol 15: e2000653, 2017.

23. Grimm D, Kay MA, and Kleinschmidt JA. Helper virusfree, optically controllable, and two-plasmid-based production of adeno-associated virus vectors of serotypes 1 to 6. Mol Ther 7: 839-850, 2003.

24. Guillozet-Bongaarts AL, Garcia-Sierra F, and Berry RW. Tau, tangles, and Alzheimer's disease. Biochim Biophys Acta 1739: 216-223, 2005.

25. Han BH, Zhou M-L, Johnson AW, Singh I, Liao F, Vellimana AK, Nelson JW, Milner E, Cirrito JR, Basak J, Yoo M, Dietrich HH, Holtzman DM, and Zipfel GJ. Contribution of reactive oxygen species to cerebral amyloid angiopathy, vasomotor dysfunction, and microhemorrhage in aged Tg2576 mice. Proc Natl Acad Sci U S A 112: E881-E890, 2015.

26. Heredia L, Helguera P, de Olmos S, Kedikian G, Solá Vigo F, LaFerla F, Staufenbiel M, de Olmos J, Busciglio J, Cáceres A, and Lorenzo A. Phosphorylation of actindepolymerizing factor/cofilin by LIM-kinase mediates amyloid beta-induced degeneration: a potential mechanism of neuronal dystrophy in Alzheimer's disease. $J$ Neurosci 26: 6533-6542, 2006.

27. Herms J and Dorostkar MM. Dendritic spine pathology in neurodegenerative diseases. Aпnu Rev Pathol 11: 221-250, 2016.

28. Ho Y-S, Xiong Y, Ho DS, Gao J, Chua BHL, Pai H, and Mieyal JJ. Targeted disruption of the glutaredoxin 1 gene does not sensitize adult mice to tissue injury induced by ischemia/reperfusion and hyperoxia. Free Radic Biol Med 43: 1299-1312, 2007.

29. Hotulainen $P$ and Hoogenraad CC. Actin in dendritic spines: connecting dynamics to function. J Cell Biol 189: 619-629, 2010.

30. Huang W, Zhu PJ, Zhang S, Zhou H, Stoica L, Galiano M, Krnjević K, Roman G, and Costa-Mattioli M. mTORC2 controls actin polymerization required for consolidation of long-term memory. Nat Neurosci 16: 441-448, 2013.

31. Hung R-J, Pak CW, and Terman JR. Direct redox regulation of F-actin assembly and disassembly by mical. Science 334: 1710-1713, 2011.

32. Hung R-J, Spaeth CS, Yesilyurt HG, and Terman JR. SelR reverses mical-mediated oxidation of actin to regulate Factin dynamics. Nat Cell Biol 15: 1445-1454, 2013.
33. Johnson WM, Wilson-Delfosse AL, Chen SG, and Mieyal JJ. The roles of redox enzymes in Parkinson's disease: focus on glutaredoxin. Ther targets Neurol Dis 2: e790, 2015.

34. Kommaddi RP, Das D, Karunakaran S, Nanguneri S, Bapat D, Ray A, Shaw E, Bennett DA, Nair D, and Ravindranath V. A $\beta$ mediates F-actin disassembly in dendritic spines leading to cognitive deficits in Alzheimer's disease. $J$ Neurosci 38: 1085-1099, 2018.

35. Lassing I, Schmitzberger F, Björnstedt M, Holmgren A, Nordlund P, Schutt CE, and Lindberg U. Molecular and structural basis for redox regulation of $\beta$-actin. $J$ Mol Biol 370: 331-348, 2007.

36. Li S, Hong S, Shepardson NE, Walsh DM, Shankar GM, and Selkoe D. Soluble oligomers of amyloid $\beta$ protein facilitate hippocampal long-term depression by disrupting neuronal glutamate uptake. Neuron 62: 788-801, 2009.

37. Martins RN, Villemagne V, Sohrabi HR, Chatterjee P, Shah TM, Verdile G, Fraser P, Taddei K, Gupta VB, RaineySmith SR, Hone E, Pedrini S, Lim WL, Martins I, Frost S, Gupta S, O'Bryant S, Rembach A, Ames D, Ellis K, Fuller SJ, Brown B, Gardener SL, Fernando B, Bharadwaj P, Burnham S, Laws SM, Barron AM, Goozee K, Wahjoepramono EJ, Asih PR, Doecke JD, Salvado O, Bush AI, Rowe CC, Gandy SE, and Masters CL. Alzheimer's disease: a journey from amyloid peptides and oxidative stress, to biomarker technologies and disease prevention strategiesgains from AIBL and DIAN cohort studies. J Alzheimers Dis 62: 965-992, 2018.

38. McBean GJ, Aslan M, Griffiths HR, and Torrão RC. Thiol redox homeostasis in neurodegenerative disease. Redox Biol 5: 186-194, 2015.

39. Mendoza-Naranjo A, Contreras-Vallejos E, Henriquez DR, Otth C, Bamburg JR, Maccioni RB, and Gonzalez-Billault C. Fibrillar amyloid- $\beta 1-42$ modifies actin organization affecting the cofilin phosphorylation state: a role for Rac1/ cdc42 effector proteins and the slingshot phosphatase. $J$ Alzheimers Dis 29: 63-77, 2012.

40. Nanguneri S, Pramod RT, Efimova N, Das D, Jose M, Svitkina T, and Nair D. Characterization of nanoscale organization of F-actin in morphologically distinct dendritic spines in vitro using supervised learning. eNeuro 6: 042518.2019, 2019.

41. Newman SF, Sultana R, Perluigi M, Coccia R, Cai J, Pierce WM, Klein JB, Turner DM, and Butterfield DA. An increase in S-glutathionylated proteins in the Alzheimer's disease inferior parietal lobule, a proteomics approach. $J$ Neurosci Res 85: 1506-1514, 2007.

42. Ouyang W, Aristov A, Lelek M, Hao X, and Zimmer C. Deep learning massively accelerates super-resolution localization microscopy. Nat Biotechnol 36: 460-468, 2018.

43. Pai H V, Starke DW, Lesnefsky EJ, Hoppel CL, and Mieyal JJ. What is the functional significance of the unique location of glutaredoxin 1 (GRx1) in the intermembrane space of mitochondria? Antioxid Redox Signal 9: 2027-2033, 2007.

44. Ray A, Sehgal N, Karunakaran S, Rangarajan G, and Ravindranath V. MPTP activates ASK1-p38 MAPK signaling pathway through TNF-dependent Trx1 oxidation in parkinsonism mouse model. Free Radic Biol Med 87: 312325, 2015.

45. Resende R, Moreira PI, Proença T, Deshpande A, Busciglio $\mathrm{J}$, Pereira $\mathrm{C}$, and Oliveira CR. Brain oxidative stress in a triple-transgenic mouse model of Alzheimer disease. Free Radic Biol Med 44: 2051-2057, 2008. 
46. Rodriguez-Rocha H, Garcia Garcia A, Zavala-Flores L, Li S, Madayiputhiya N, and Franco R. Glutaredoxin1 protects dopaminergic cells by increased protein glutathionylation in experimental Parkinson's disease. Antioxid Redox Signal 17: 1676-1693, 2012.

47. Rohr U-P, Heyd F, Neukirchen J, Wulf M-A, Queitsch I, Kroener-Lux G, Steidl U, Fenk R, Haas R, and Kronenwett R. Quantitative real-time PCR for titration of infectious recombinant AAV-2 particles. J Virol Methods 127: 40-45, 2005.

48. Rubenstein PA and Wen K-K. NATure of actin aminoterminal acetylation. Proc Natl Acad Sci U S A 115: 43144316, 2018.

49. Sabens EA, Distler AM, and Mieyal JJ. Levodopa deactivates enzymes that regulate thiol-disulfide homeostasis and promotes neuronal cell death: implications for therapy of Parkinson's disease. Biochemistry 49: 2715-2724, 2010.

50. Saeed U, Durgadoss L, Valli RK, Joshi DC, Joshi PG, and Ravindranath V. Knockdown of cytosolic glutaredoxin1 leads to loss of mitochondrial membrane potential: implication in neurodegenerative diseases. PLoS One 3: e2459, 2008.

51. Saeed U, Ray A, Valli RK, Kumar AMR, and Ravindranath V. DJ-1 loss by glutaredoxin but not glutathione depletion triggers daxx translocation and cell death. Antioxid Redox Signal 13: 127-144, 2010.

52. Sakai J, Li J, Subramanian KK, Mondal S, Bajrami B, Hattori H, Jia Y, Dickinson BC, Zhong J, Ye K, Chang CJ, Ho Y-S, Zhou J, and Luo HR. Reactive oxygen speciesinduced actin glutathionylation controls actin dynamics in neutrophils. Immunity 37: 1037-1049, 2012.

53. Shelton MD, Chock PB, and Mieyal JJ. Glutaredoxin: role in reversible protein s-glutathionylation and regulation of redox signal transduction and protein translocation. Antioxid Redox Signal 7: 348-366, 2005.

54. Spires-Jones TL, Meyer-Luehmann M, Osetek JD, Jones PB, Stern EA, Bacskai BJ, and Hyman BT. Impaired spine stability underlies plaque-related spine loss in an Alzheimer's disease mouse model. Am J Pathol 171: 1304-1311, 2007.

55. Spires TL, Meyer-Luehmann M, Stern EA, McLean PJ, Skoch J, Nguyen PT, Bacskai BJ, and Hyman BT. Dendritic spine abnormalities in amyloid precursor protein transgenic mice demonstrated by gene transfer and intravital multiphoton microscopy. J Neurosci 25: 7278-7287, 2005.

56. Stapper ZA and Jahn TR. Changes in glutathione redox potential are linked to $\mathrm{A} \beta 42$-induced neurotoxicity. Cell Rep 24: 1696-1703, 2018.

57. Steger C. An unbiased detector of curvilinear structures carsten steger. IEEE Trans Pattern Anal Mach Intell 20: 113-125, 1998.

58. Stojkov D, Amini P, Oberson K, Sokollik C, Duppenthaler A, Simon H-U, and Yousefi S. ROS and glutathionylation balance cytoskeletal dynamics in neutrophil extracellular trap formation. J Cell Biol 216: 4073-4090, 2017.

59. Sultana R, Perluigi M, and Butterfield DA. Lipid peroxidation triggers neurodegeneration: a redox proteomics view into the Alzheimer disease brain. Free Radic Biol Med 62: 157-169, 2013.

60. Sultana R, Reed T, Perluigi M, Coccia R, Pierce WM, and Butterfield DA. Proteomic identification of nitrated brain proteins in amnestic mild cognitive impairment: a regional study. J Cell Mol Med 11: 839-851, 2007.

61. Terman JR and Kashina A. Post-translational modification and regulation of actin. Curr Opin Cell Biol 25: 30-38, 2013.
62. Tönnies E and Trushina E. Oxidative stress, synaptic dysfunction, and Alzheimer's disease. J Alzheimers Dis 57: 1105-1121, 2017.

63. Towne $\mathrm{C}$ and Aebischer P. Lentiviral and adeno-associated vector-based therapy for motor neuron disease through RNAi. Methods Mol Biol 555: 87-108, 2009.

64. Towne C, Raoul C, Schneider BL, and Aebischer P. Systemic AAV6 delivery mediating RNA interference against SOD1: neuromuscular transduction does not alter disease progression in fALS mice. Mol Ther 16: 1018-1025, 2008.

65. Tsai J, Grutzendler J, Duff K, and Gan W-B. Fibrillar amyloid deposition leads to local synaptic abnormalities and breakage of neuronal branches. Nat Neurosci 7: 1181-1183, 2004.

66. Wang H, Ma J, Tan Y, Wang Z, Sheng C, Chen S, and Ding J. Amyloid- $\beta 1-42$ induces reactive oxygen speciesmediated autophagic cell death in U87 and SH-SY5Y cells. J Alzheimers Dis 21: 597-610, 2010.

67. Wang Z-X, Tan L, Liu J, and Yu J-T. The essential role of soluble A $\beta$ oligomers in alzheimer's disease. Mol Neurobiol 53: 1905-1924, 2016.

68. Wilson $\mathrm{C}$ and González-Billault C. Regulation of cytoskeletal dynamics by redox signaling and oxidative stress: implications for neuronal development and trafficking. Front Cell Neurosci 9: 381, 2015.

69. Xu K, Zhong G, and Zhuang X. Actin, spectrin, and associated proteins form a periodic cytoskeletal structure in axons. Science 339: 452-456, 2013.

70. Zhang C, Rodriguez C, Spaulding J, Aw TY, and Feng J. Age-dependent and tissue-related glutathione redox status in a mouse model of Alzheimer's disease. J Alzheimers Dis 28: 655-666, 2012.

Address correspondence to: Dr. Reddy Peera Kommaddi Centre for Neuroscience Indian Institute of Science C.V. Raman Avenue Bangalore 560012 India

E-mail: reddy@iisc.ac.in

Date of first submission to ARS Central, February 21, 2019; date of final revised submission, October 9, 2019; date of acceptance, October 13, 2019.

\begin{tabular}{|c|}
\hline $\begin{aligned} & \text { Abbreviations Used } \\
\mathrm{A} \beta & =\text { amyloid beta } \\
\mathrm{AAV}= & \text { adeno-associated virus } \\
\mathrm{AD}= & \text { Alzheimer's disease } \\
\mathrm{ADL}= & \text { adolescent } \\
\mathrm{aMCI}= & \text { amnestic mild cognitive impairment } \\
\mathrm{AMS}= & 4 \text {-acetamido-4'-maleimidylstilbene- } \\
& 2,2^{\prime} \text {-disulfonic acid }, \text { disodium salt } \\
\text { ANNA-PALM }=\text { artificial neural network accelerated- } & \text { photoactivation localization } \\
& \text { microscopy } \\
\mathrm{ANOVA}= & \text { analysis of variance } \\
\mathrm{APP} / \mathrm{PS} 1= & \text { amyloid precursor protein/presenilin } 1 \\
\text { double mutant } & \text { BCA }=\text { bicinchoninic acid }\end{aligned}$ \\
\hline
\end{tabular}




$\begin{aligned} & \text { Abbreviations Used (Cont.) } \\ & \mathrm{BSA}=\text { bovine serum albumin } \\ & \mathrm{cFC}=\text { contextual fear conditioning } \\ & \mathrm{DAPI}=4^{\prime}, 6 \text {-diamidino-2-phenylindole } \\ & \mathrm{DCF}=2^{\prime}, 7^{\prime} \text {-dichlorofluorescein } \\ & \mathrm{DIV}=\text { day-in-vitro } \\ & \mathrm{dSTORM}=\text { direct stochastic optical reconstruction } \\ & \text { microscopy } \\ & \mathrm{Grx} 1=\text { glutaredoxin } 1 \\ & \mathrm{GSH}=\text { glutathione } \\ & \mathrm{H}_{2} \mathrm{DCFDA}=2^{\prime}, 7^{\prime} \text {-dichlorodihydrofluorescein diacetate } \\ & \mathrm{IP}=\text { immunoprecipitation } \\ & \mathrm{MA}=\text { middle aged } \\ & \mathrm{PBS}=\text { phosphate-buffered saline }\end{aligned}$

$\begin{aligned} \text { PNS } & =\text { postnuclear supernatant } \\ \text { PrSH } & =\text { protein thiol } \\ \text { PrSSG } & =\text { glutathionylated proteins } \\ \text { PSD95 } & =\text { postsynaptic density protein } 95 \\ \text { PVDF } & =\text { polyvinylidene difluoride } \\ \text { qPCR } & =\text { quantitative polymerase chain reaction } \\ \text { ROS } & =\text { reactive oxygen species } \\ \text { SDS-PAGE } & =\text { sodium dodecyl sulfate/polyacrylamide } \\ \text { SEM } & =\text { gtandard electrophoresis } \\ \text { SNAP29 } & =\text { synaptosomal-associated } \\ \text { protein } 29 \mathrm{kDa} & \text { proan } \\ \text { TGX } & =\text { tris-glycine-extended } \\ \mathrm{Trx} & =\text { thioredoxin } \\ \mathrm{WT} & =\text { wild type }\end{aligned}$

